\title{
Transforming the Snapdragon Aurone Biosynthetic Genes into Petunia Alters Coloration Patterns in Transgenic Flowers
}

\author{
Chen-Kuen Wang, Yi-Chin Chin, Chih-Yu Lin, Po-Yen Chen, Kin-Ying To* \\ Agricultural Biotechnology Research Center, Academia Sinica, Taiwan \\ Email: "kyto@gate.sinica.edu.tw \\ Received 17 November 2015; accepted 14 December 2015; published 18 December 2015 \\ Copyright (C) 2015 by authors and Scientific Research Publishing Inc. \\ This work is licensed under the Creative Commons Attribution International License (CC BY). \\ http://creativecommons.org/licenses/by/4.0/

(c) $\underset{\mathrm{BY}}{\mathrm{i}}$ Open Access

\begin{abstract}
Aurones belong to a small class of flavonoids that provide yellow color in some floricultural plants including snapdragon. To explore novel flower coloration, two full-length cDNAs encoding chalcone 4'-O-glucosyltransferase (designated as $S R Y 4^{\prime} C G T$ ) and aureusidin synthase (designated as $S R Y A S 1)$ in the aurone biosynthetic pathway were cloned from yellow flowers of snapdragon (Antirrhinum majus cv. Ribbon Yellow). Binary vectors were constructed and transformed separately into Petunia hybrida harboring blue flowers. Only a few flowers in 4 out of 9 transgenic SRY4'CGT plants showed variegated blue-white sectors; as time passed, amounts of variegated flowers and proportion of white sectors in the background blue color of the new-born flowers gradually increased, until finally, the petal color was completely white in all late-born flowers. In contrast, a few flowers in 3 out of 13 transgenic SRYAS1 plants showed variegated blue-white sectors; but, the amounts of variegated flowers did not increase over the whole flowering stage, and no complete white flowers were observed. RNA samples isolated from blue and white sectors of $T_{1}$ transgenic SRY4'CGT plants were analyzed by reverse transcription-PCR, transgenic SRY4'CGT transcripts were detected in both sectors; however, transcripts of an upstream gene, chalcone synthase ( $\mathrm{CHS}$ ), were abundantly detected in the blue sectors but largely reduced in the white sectors, suggesting that the expression of $\mathrm{CHS}$ gene was suppressed in white sectors of transgenic plants. Furthermore, HPLC coupled with mass spectrometry demonstrated cyandin, malvidin and their derivatives were absent in white sectors, causing the white phenotype. Our findings may be attractive to molecular breeders.
\end{abstract}

\section{Keywords}

Agrobacterium-Mediated Transformation, Aurone, Compound Identification, Flower Color, Genetic Engineering, HPLC Profiling, Suppression, Transgene-Induced Gene Silencing

\footnotetext{
${ }^{*}$ Corresponding author.
}

How to cite this paper: Wang, C.-K., Chin, Y.-C., Lin, C.-Y., Chen, P.-Y. and To, K.-Y. (2015) Transforming the Snapdragon Aurone Biosynthetic Genes into Petunia Alters Coloration Patterns in Transgenic Flowers. Advances in Bioscience and Biotechnology, 6, 702-722. http://dx.doi.org/10.4236/abb.2015.612073 


\section{Introduction}

Flower color is an important feature in floricultural plants, contributing to the major value in the flower market. In general, flower color is determined by three classes of pigments: flavonoids, carotenoids and betalains. Flavonoids are the major contributors in the determination of a range of colors such as yellow, orange, red and purple; and the carotenoids are the red, orange and yellow lipid-soluble pigments found embedded in the membranes of choroplasts and chromoplasts [1] [2]. The third class of pigments, betalains, can be found only in certain plant species of the Carophyllales and in some higher fungi such as fly agaric [2] [3]. Over the past few decades, almost all the key genes in the main biosynthesis pathways of flavonoids and carotenoids have been cloned from a range of plant species, and genetic engineering has been carried out to breed new cultivars with novel flower colors in many floricultural plants [2]-[8]. Some branch/modifying enzymes are limited to certain plant species; however, most of these genes are not yet known.

Flavonoids, starting from the general phenylpropanoid pathway, can be subdivided into several major groups according to their structures: chalcones, aurones, isoflavonoids, flavones, flavonols, flavan-3-ols, anthocyanins, condensed tannins (proanthocyanins) and phlobaphenes [3] [9]. Among these groups, anthocyanins, flavonols, chalcones and aurones contribute color to plants. Aurones are the bright yellow pigments found in some ornamental flowers such as snapdragon (Antirrhinum majus), cosmos and coreopsis [10]. Biochemical and flavonoid mutant studies from A. majus clearly revealed that aurones were produced from chalcones [4] [10]. The first common flavonoid compound is 2',4',6',4-tetrahydroxychalcone (THC) or also known as naringenin chalcone, which is the catalytic product of the key enzyme chalcone synthase (CHS). In many floricultural plants, THC is converted into naringenin by chalcone isomerase (CHI), and naringenin is further converted into different kinds of anthocyanins, flavonols and flavones by several enzymes in the flavonoid biosynthesis pathway. In yellowflowering plants such as snapdragon, THC is converted into THC 4'-O-glucoside by UDP-glucose: chalcone 4'-O-glucosyltransferase (4'CGT), and THC 4'-O-glucoside is further converted into aureusidin 6-O-glucoside (yellow pigment) by aureusidin synthase (AS) [11]. In addition, in vitro activity of AS, catalyzing THC into aureusidin (yellow pigment), has also been reported [10]. Both genes, designated as AmAS1 and Am4'CGT, have been cloned from yellow snapdragon (A. majus cv. Yellow Butterfly) and it has been demonstrated that both genes are essential for aurone biosynthesis and yellow coloration [10] [11]. Subsequently, coexpression of the AmAS1 and Am4'CGT transgenes together with suppression of endogenous anthocyanin biosynthetic gene [flavanone 3-hydroxylase $(F 3 H)$ or dihydroflavonol 4-reductase $(D F R)]$ produced yellow flowers in transgenic lines of Torenia hybrid cv. Summer Wave Blue, a cultivar has blue flowers [11].

We are interested in molecular breeding of flower color since flower color is one of the most attractive characteristics in garden ornamentals and contributes to the major value in the floricultural markets [2] [12]. Petunia is one of the most popular ornamentals worldwide, and has been used as one of the model plants for various studies including flavonoid biosynthesis, floral development, male sterility, self-incompatibility, retroelement activity, transposons, epigenetics, transformation, senescence, and so on [13] [14]. In this study, we try to generate bright yellow flowers in petunia. As a first step to achieve our goal, here we report the cloning and construction into expression vectors of AS1 and 4'CGT cDNAs from yellow flowers in another snapdragon cultivar, Antirrhinum majus cv. Ribbon Yellow. These constructs were individually introduced into Petunia hybrida harboring blue flowers by using Agrobacterium-mediated method. Although yellow flowers were not observed; however, a few flowers in several transgenic plants showed variegated blue-white sectors and attractive coloration patterns. Here we just summarize what we have done and observed in single gene transformation. Breeding transgenic plants coexpressing both transgenes are in progress. The findings reported herein will be useful for breeding new cultivars with attractive coloration patterns.

\section{Materials and Methods}

\subsection{Plant Materials}

Seeds of Antirrhinum majus cv. Ribbon Yellow and Petunia hybridacv. Extra Blue were purchased from KnownYou Seed Company, Taiwan. For gene cloning, seeds of A. majus were germinated, and young seedlings were transferred into pots and grown in a greenhouse until maturity. For plant transformation, seeds of $P$. hybrida were sterilized with $1 \%$ sodium hypochlorite for $20 \mathrm{~min}$, washed thoroughly with sterile water, and germinated on MS basal medium containing MS salts [15], $2 \%$ sucrose, $0.8 \%$ bacto-agar, $\mathrm{pH}$ 5.7. The cultures were then 
incubated in a $26^{\circ} \mathrm{C}$ growth chamber under a 16 -h illumination $\left(100 \mu \mathrm{mol} / \mathrm{m}^{2} / \mathrm{sec}\right)$ and 8 -h dark cycle. In vitro explants grown for 2 to 3 weeks were used to establish the plant transformation system.

\subsection{Gene Cloning and Construction of Plant Expression Vector}

To clone the aurone biosynthetic genes, RNA from different developmental stages of yellow flowers of A. majus was isolated by TRIzol protocol as previously described [16]. Primers SRY4'CGT-F1 (5'-ATGGGAGAAGAATACAAGAAA-3') and SRY4'CGT-R1 (5'-TTAACGAGTGACCGAGTTGAT-3') specific for A. majus chalcone 4'-O-glucosyltransferase (4'CGT), and primers SRYAS1-F1 (5'-ATGTTCAAAAATCCTAATATC-3') and SRYAS1-R1 (5'-TTAGCCATCAAGCTCAATCTT-3') specific for A. majus aureusidin synthase (AS1) were synthesized to amplify full-length reading frames of SRY4'CGT (1374 bp) and SRYAS1 (1689 bp), respectively. RT-PCR was carried out as previously described [16]. PCR products were analyzed by $1 \%$ agarose gel electrophoresis. After purification, PCR products were cloned into pGEM-T Easy vector (Promega), and then sequenced by an automatic DNA sequencer (ABI PRISM 3730 DNA sequencer, Perkin-Elmer). To construct the expression vectors, PCR products of snapdragon 4'CGT and AS1 were cloned into Gateway binary vectors pH2GW7 (carrying the hygromycin selection marker) and pK2WG7 (carrying the kanamycin selection marker), respectively, by using the pCR8/GW/TOPO TA cloning kit (Invitrogen). The resulting vectors pSRY4'CGT and pSRYAS1 were isolated, screened by PCR analysis, and confirmed by DNA sequencing analysis.

\subsection{Agrobacterium-Mediated Transformation}

To introduce the vector DNA into Agrobacterium, $50 \mu \mathrm{l}$ competent cells of Agrobacterium tumefaciens strain GV3101 were mixed with 100 ng plasmid DNA (pSRY4'CGT or pSRYAS1) and incubated on ice for 1 to 2 min. The mixture was then transferred into an ice-cold $0.1-\mathrm{cm}$ electroporation cuvette. Electroporation was carried out in a Bio-Rad Gene Pulser II (USA) using the following parameters: $25 \mu \mathrm{F}, 200 \Omega$, and $1.25 \mathrm{kV}$. After electroporation, $1 \mathrm{ml}$ of YEP medium (10 g/l peptone; $10 \mathrm{~g} / \mathrm{l}$ yeast extract; $5 \mathrm{~g} / \mathrm{l} \mathrm{NaCl}$; $\mathrm{pH}$ 7.0) was added immediately into the mixture, incubated at $28^{\circ} \mathrm{C}$ for $2 \mathrm{~h}$, and $200 \mu \mathrm{l}$ of bacterial mixture was transferred onto selective YEP plates containing $100 \mathrm{mg} / \mathrm{l}$ spectinomycin. The plates were incubated at $28^{\circ} \mathrm{C}$ for 2 days. A single colony of plasmid-carrying Agrobacterium was picked up and incubated in $4 \mathrm{ml}$ YEP medium containing $100 \mathrm{mg} / \mathrm{l}$ spectinomycin at $28^{\circ} \mathrm{C}$ overnight. The value of $\mathrm{OD}_{600}$ value should be $0.1-0.3$. One $\mathrm{ml}$ of overnight culture was

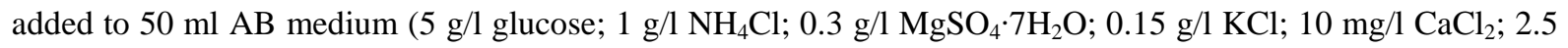
$\mathrm{mg} / \mathrm{l} \mathrm{FeSO}{ }_{4} \cdot 7 \mathrm{H}_{2} \mathrm{O} ; 3 \mathrm{~g} / \mathrm{l} \mathrm{K}_{2} \mathrm{HPO}_{4} ; 1.15 \mathrm{~g} / \mathrm{N} \mathrm{NaH}_{2} \mathrm{PO}_{4} \cdot \mathrm{H}_{2} \mathrm{O}$ ) supplemented with $100 \mathrm{mg} / \mathrm{l}$ spectinomycin, and incubated for 1 day at $28^{\circ} \mathrm{C}$. Acetosyringone (Fluka Chemika, Switerland) and glucose were added to the $\mathrm{AB} /$ spectinomycin medium to yield a final concentration of $100 \mu \mathrm{M}$ and $5 \%$, respectively, and the culture was further incubated $4 \mathrm{~h}$ at $28^{\circ} \mathrm{C}$. The bacterial culture was then centrifuged at $5000 \mathrm{rpm}$ for $5 \mathrm{~min}$, and the pellet was dissolved in KCMS medium (MS salts; $0.9 \mathrm{mg} / \mathrm{l}$ thiamine; $0.2 \mathrm{mg} / \mathrm{l}$ 2,4-dichlorophenylacetic acid (2,4-D); 200 $\mathrm{mg} / \mathrm{l} \mathrm{KH}_{2} \mathrm{PO}_{4}$; $\mathrm{pH}$ 5.6) supplemented with $100 \mu \mathrm{M}$ acetosyringone and $5 \%$ glucose. The suspension was then diluted such that the final $\mathrm{OD}_{600}$ reading was $0.8-1.0$.

Agrobacterium-mediated transformation was carried out as previously described [17] with modifications. For pre-culture prior to Agrobacterium infection, cotyledons or leaves were excised from 2- to 3-week-old in vitro plantlets of $P$. hybrida one day before infection, and incubated in KCMS solid medium supplemented with 100 $\mu \mathrm{M}$ acetosyringone for one day. Infection was carried out by adding $15 \mathrm{ml}$ of diluted Agrobacterium suspension to the pre-cultured explants for $1 \mathrm{~h}$. Excess Agrobacterium was blotted from the explants on sterile filter papers, then the infected explants were transferred onto KCMS solid medium containing $100 \mu \mathrm{M}$ acetosyringone and $5 \%$ glucose, and left to grow for 2 days in a dark growth chamber at $26^{\circ} \mathrm{C}$. Explants were then washed 2-3 times with shaking (the first shaking was at $220 \mathrm{rpm}$, and subsequent shakings were at $100 \mathrm{rpm}, 30 \mathrm{~min}$ each time) in KCMS liquid medium supplemented with $200 \mathrm{mg} / \mathrm{l}$ timentin. Explants were dried with sterile filter papers, and transferred to MSZ medium (MS salts; Nitsch vitamins; $1 \%$ sucrose; $2 \%$ mg/l zeatin; 0.7\% bacto-agar; pH 6.0) supplemented with $200 \mathrm{mg} / \mathrm{l}$ timentin to inhibit Agrobacterium growth and $10 \mathrm{mg} / \mathrm{l}$ hygromycin (for selection in transforming vector pSRY4'CGT) or $100 \mathrm{mg} / \mathrm{l}$ kanamycin (for selection in transforming vector pSRYAS1), and incubated at $26^{\circ} \mathrm{C}$ in a growth chamber with a 16-h light $\left(100-200 \mu \mathrm{mol} / \mathrm{m}^{2} / \mathrm{sec}\right)$ and 8-h dark cycle. Explants were transferred to a new MSZ medium supplemented with $200 \mathrm{mg} / \mathrm{l}$ timentin and suitable antibiotic $(10 \mathrm{mg} / \mathrm{l}$ hygromycin or $100 \mathrm{mg} / \mathrm{l}$ kanamycin) every two weeks. Formation of the initial calli could be seen on the wound 
site of explants after 3 weeks of transformation, and shoots were regenerated after 6 weeks. Single shoot was excised from the callus and transferred onto the MS basal medium supplemented with $100 \mathrm{mg} / \mathrm{l}$ timentin. The rooted plantlets were transferred into pots and grown in a greenhouse.

\subsection{Reverse Transcription-PCR Analysis}

Total RNA was extracted from transgenic flowers, treated with DNase I, and 100 ng of treated RNA was used to perform reverse transcription-PCR (RT-PCR) analysis as previously described [12]. For amplification of genespecific region, primers SRYAS1-F1 (5'-ATGTTCAAAAATCCTAATATC-3') and SRYAS1-R2 (5'-ACCAGGTGAATCATAGTTCCA-3') were employed to amplify the 624-bp-long DNA fragment of snapdragon AS1 (GenBank accession no. EF650014), primers SRY4'CGT-F2 (5'-ATGGGAGAAGAATACAAGAAAAC-3') and SRY4'CGT-R3 (5'-CATGCTGGTGGATACTTC-3') were employed to amplify the 390-bp-long DNA fragment of snapdragon 4'CGT (GenBank accession no. EF650015), and primers CHS-C1 (5'-ACAACAAGGGCGCTCGAG-3') and CHS-C2 (5'-CAAGCCCTTCACCAGTAG-3') were employed to amplify the 558-bp-long DNA fragment of Petunia chalcone synthase (GenBank accession No. AF233638).

\subsection{Seedling Assay for Kanamycin or Hygromycin Resistance}

$\mathrm{T}_{1}$ seeds from self-fertilized transgenic plants were sterilized with $1 \%$ sodium hypochlorite as mentioned above. They were germinated in MS basal medium supplemented with $10 \mathrm{mg} / \mathrm{l}$ hygromycin (for transgenic SRY4'CGT lines) or $100 \mathrm{mg} / \mathrm{l}$ kanamycin (for transgenic SRYAS1 lines) for 3 weeks. Seedlings with white or pale green cotyledons, no true leaf development, inhibition of root extension and plant development were considered to be hygromycin or kanamycin sensitive, while seedlings with green cotyledons and healthy development of leaves and roots were considered to be hygromycin or kanamycin resistant.

\subsection{Sample Preparation}

Freeze-dried petals from transgenic Petunia flowers carrying SRY4'CGT or SRYAS1 transgene were homogenized by using liquid nitrogen with a pestle and mortar, transferred into a tube and dissolved in $50 \%$ acetonitrile containing $0.1 \%$ (vol/vol) trifluoroacetic acid (TFA) to a final concentration of $0.1 \mathrm{~g} / \mathrm{ml}$ (fresh weight $/ \mathrm{vol}$ ). The mixture was rotated for $3 \mathrm{~h}$ at room temperature, and then briefly centrifuged. The supernatant was transferred into a clean tube, and then vacuum-dried. The extracts were resuspended in $50 \%$ acetonitrile containing $0.1 \%$ (vol/vol) formic acid (FA), the final concentration was adjusted to $0.1 \mathrm{~g} / \mathrm{ml}$ (fresh weight), and used for HPLCDAD-MS/MS analysis.

\subsection{HPLC-DAD-MS/MS Analysis}

The Agilent 1100 series liquid chromatograph system (Agilent, Waldbronn, Germany) coupled with a diodearray detector (DAD) and a Thermo Finnigan LCQ Advantage ion-trap mass spectrometer (San Jose, CA, USA) was employed for analysis at the Metabolomics Core Facility of the Agricultural Biotechnology Research Center, Academia Sinica, Taiwan. The extracts were separated by HPLC with a RP-C18 column (Phenomenex Luna $3 \mu \mathrm{m} \mathrm{C} 18,150 \mathrm{~mm} \times 2.0 \mathrm{~mm}$ ) at a flow rate of $0.2 \mathrm{ml} / \mathrm{min}$. The mobile phase consisted of water containing $0.1 \%(\mathrm{v} / \mathrm{v})$ FA (mobile phase A) and acetonitrile containing $0.1 \%(\mathrm{v} / \mathrm{v})$ FA (mobile phase $\mathrm{B}$ ). The following conditions were used for the gradient elution: 10\% - 23\% B for 3 min, next with 23\% B for 17 min, 23\% - 80\% B for 2 min and then $80 \%$ B for $3 \mathrm{~min}$. The sample injection volume was $10 \mu \mathrm{l}$. Eluting peaks were monitored by DAD under the following conditions: Chalcones, aurones, anthocyanidins, and flavones were monitored respectively at $360 \mathrm{~nm}$, at $400 \mathrm{~nm}$, at $520 \mathrm{~nm}$, and at $360 \mathrm{~nm}$ [11]. Both positive and negative ion polarity modes were used for compound ionization. The MS optimized parameters in positive ion mode were as follows: source voltage, $4.5 \mathrm{kV}$; sheath gas $\left(\mathrm{N}_{2}\right), 50$ arbitrary units; capillary temperature, $250^{\circ} \mathrm{C}$; capillary voltage, $3 \mathrm{~V}$; tube lens offset voltage, $30 \mathrm{~V}$; in the negative ion mode, source voltage, $-4 \mathrm{kV}$; capillary voltage, $-47 \mathrm{~V}$; tube lens offset voltage, $-45 \mathrm{~V}$. For full scan MS analysis, spectra were recorded in the range of $\mathrm{m} / \mathrm{z} 100$ - 2000.The data-de- pendent program was set so that the three most intense ions in each survey scan were selected and subjected to tandem mass spectrometry. The HPLC-DAD-MS/MS system was controlled by Xcalibur 2.0 SR2 software (Thermo Finnigan, San Jose, CA, USA). 


\section{Results}

\subsection{Cloning and Sequence Analysis of Full-Length cDNAs in the Aurone Biosynthetic Pathway from Yellow Snapdragon Flowers}

Yellow flowers of snapdragon (Antirrhinum majus cv. Ribbon Yellow) at different stages from tiny flower bud to mature flower (Figure 1) were harvested, total RNA was isolated, and RT-PCR was carried out to clone the full-length cDNAs encoding chalcone 4'-O-glucosyltransferase (designated as SRY4'CGT) and aureusidin synthase (designated as SRYAS1) in aurone biosynthetic pathway. The SRY4'CGT clone is 1374 bp in length and contains a reading frame of 457 amino acids. Our clone is the same length as the published A. majus Am4'CGT nucleotide sequence ([11]; GenBank accession No. AB198665); however, it differed from the published clone by 16 nucleotides and 8 amino acids (Figure 2). To examine the accuracy of the sequence of our SRY4'CGT cDNA clone, the corresponding genomic DNA fragment was amplified by PCR analysis using genomic DNA isolated from A. majus cultivar Ribbon Yellow and primer set (SRY4'CGT-F1 and SRY4'CGT-R1) and then sequenced. As shown in Figure 2, nucleotide sequences of our cDNA and the genomic clones were identical, indicating that the genomic $S R Y 4^{\prime} C G T$ gene has no intron. This result is consistent with the finding that there is no intron in the $A m 4^{\prime} C G T$ gene of $A$. majus [11]. The differences between the published clone and our clone reflect the two different cultivars (Ribbon Yellow versus Yellow Butterfly) examined. The full-length cDNA and genomic DNA encoding SRY4'CGT in cultivar Ribbon Yellow were submitted to GenBank database with accession numbers EF650015 and JQ234673, respectively. It was reported that chalcone 4'-O-glucosyltransferase catalyzes 2',4',6', 4-tetrahydroxychalcone (THC) to THC 4'-O-glucoside (colorless) or 2',4',6',3,4-pentahydroxychalcone (PHC) to PHC 4'-O-glucoside (colorless) in aurone biosynthetic pathway [11].

The other clone, SRYAS1, is 1689 bp in length and contains a reading frame of 562 amino acids. Our SRYAS1 differed from the published snapdragon AmAS1 nucleotide sequence ([10]; GenBank accession no. AB044884) by two bases in the coding region at nucleotide positions 285 and 892; however, the amino acids were identical to the corresponding published AmAS1 protein sequence (Figure 3). The full-length cDNA encoding SRYAS1 in snapdragon cultivar Ribbon Yellow was submitted to GenBank database with accession number EF650014. It was reported that aureusidin synthase catalyzes THC 4'-O-glucoside (colorless) or PHC 4'-Oglucoside (colorless) to produce aureusidin 6-O-glucoside (yellow pigment) or bracteatin 6-O-glucoside (yellow pigment), respectively, in the aurone biosynthetic pathway [11]. The deduced amino acid sequence of SRYAS1 protein showed high sequence similarity to the plant polyphenol oxidase family [10], which has been demonstrated to be responsible for flower coloration [10] [11] [18].

The relevant cDNA fragments were cloned separately into Gateway vectors, obtaining the expression vectors pSRY4'CGT and pSRYAS1 (Figure 4), which were confirmed by restriction enzyme digestion and DNA sequencing.

\subsection{Isolation and Phenotypic Observation of Transgenic Petunia Plants Carrying SRY4'CGT or SRYAS1 Transgene}

Agrobacterium-mediated transformation was carried out to transform pSRY4'CGT and pSRYAS1 separately into $P$. hybrida. Finally, 9 putative transgenic plants were obtained from a total of 186 cotyledon explants by

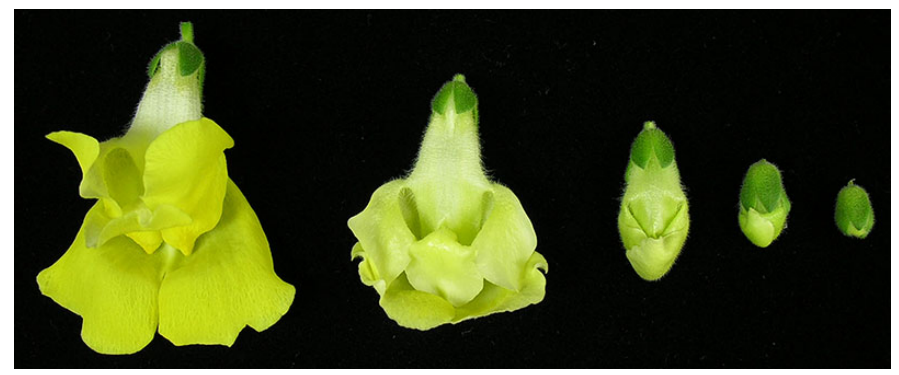

Figure 1. Flower phenotype of snapdragon. Seeds of snapdragon (Antirrhinum majus cv. Ribbon Yellow) were germinated, and young seedlings were transferred into pots and grown in greenhouse until maturity. RNA was isolated from different stages of tiny flower bud to mature flower. 
AB198665 ATGGGAGAAG AATACAAGAA AACACACACA ATAGTCTTTC ACACTTCAGA AGAACACCTC AACTCTTCAA TAGCCCTTGC AAAGTTCATA ACCAAACACC BF 650015 ATGGGAGAAG AATACAAGAA AACACACACA ATAGTCTTTC ACACTTCAGA AGAACACCTC AACTCTTCAA TAGCCCTTGC AAAGTTCATA ACCAAACACC JQ234673 ATGGGAGAAG AATACAAGAA AACACACACA ATAGTCTTTC ACACTTCAGA AGAACACCTC AACTCTTCAA TAGCCCTTGC AAAGTTCATA ACCAAACACC

AB198665 ACTCTTCAAT CTCCATCACT ATCATCAGCA CTGCCCCCGC CGAATCTTCT GAAGTGGCCA AAATTATTAA TAATCCGTCA ATAACTTACC GCGGCCTCAC BF650015 ACTCTTCAAT CTCCATCACT ATCATCAGCA CTGCCCCCGC CGAATCTTCT GAAGTGGCCA AAATTATTAA TAATCCGTCA ATAACTTACC GCGGCCTCAC JQ234673 ACTCTTCAAT CTCCATCACT ATCATCAGCA CTGCCCCCGC CGAATCTTCT GAAGTGGCCA AAATTATTAA TAATCCGTCA ATAACTTACC GCGGCCTCAC

AB198665 CGCGGTAGCG CTCCCTGAAA ATCTCACCAG TAACATTAAT AAAAACCCCG TCGAACTTTT CTTCGAAATC CCTCGTCTAC AAAACGCCAA CCTTCGAGAG BF650015 CGCGGTAGCG CTCCCTGAAA ATCTCACCAG CAACATTAAT AAAAACCCCG TCGAACTTTT CTTCGAAATC CCTCGTCTAC AAAACGCCAA CCTTGGAGAG

AB198665 GCTTTACTAG ATATTTCGCG AAAATCCGAT ATCAAAGCAT TAATCATCGA TTTCTTCTGC AATGCGGCAT TTGAAGTATC CACCAGCATG AACATACCCA BE650015 GCTTTACTAG ATATTTCGCG AAAATCCGAT ATCAAAGCAT TAATCATCGA TTTCTTCTGC AATGCGGCAT TTGAAGTATC CACCAGCATG AACATACCCA

AB198665 CTTACTTCGA CGTCAGTGGC GGCGCTTTTC TCCTCTGCAC GTTTCTCCAC CACCCGACAC TACACCAAAC TGTTCGTGGA GACATTGCGG ATTTGAACGA BF650015 CTTACTTCGA CGTCAGTGGC GGCGCTTTTC TCCTCTGCAC GTTTCTCCAC CACCCGACAC TACACCAAAC TGTTCGTGGA GACATTGCGG ATTTGAACGA JQ234673 CTTACTTCGA CGTCAGTGGC GGCGCTTTTC TCCTCTGCAC GTTTCTCCAC CACCCGACAC TACACCAAAC TGTTCGTGGA GACATTGCGG ATTTGAACGA

AB198665 TTCTGTTGAG ATGCCCGGGT TCCCATTGAT TCACTCCTCT GATTTACCAA TGAGTTTGTT TTATCGTAAG ACTAATGTTT ACAAACACTT TCTAGACACT EE650015 TTCTGTTGAG ATGCCCGGGT TCCCATTAAT TCACTCCTCT GATTTACCAA TGAGTTTGTT TTATCGTAAG AGTAATGTTT ACAAACACTT TCTAGACACI JQ234673 TTCTGTTGAG ATGCCCGGGT TCCCATTAAT TCACTCCTCT GATTTACCAA TGAGTTTGTT TTATCGTAAG AGTAATGTTT ACAAACACTT TCTAGACACT

AB198665 TCCTTAAACA TGCGCAAATC GAGTGGGATA CTCGTGAACA CGTTTGTTGC GCTCGAGTTT CGAGCTAAGG AAGCTTTGTC CAACGGTTTG TACGGTCCAA BE650015 TCCTTAAACA TGCGCAAATC GAGTGGGATA CTCGTGAACA GGTTTGTTGC GCTCGAGTTT CGAGCTAAGG AAGCTTTGTC CAACGGTTTTG TACGGTCCAA JF 3015 TCCTIAAACA TGCGCAAATC GAGIGGGATA CTG

AB198665 CTCCGCCTCT TTATTTACTT TCACATACAA TTGCCGAACC CCACGACACT AAAGTGTTGG TAAACCAACA CGAATGCCTA TCATGGCTTG ATTTGCAGCC $\begin{array}{lll}\text { BF } 650015 & \text { CTCCGCCTGT TTATTTACTT TCACATACAA TTGCCGAACC CCACGACACT AAAGTGTTGG TAAACCAACA CGACTGCCTA TCATGGCTTG ATTTGCAGCC } \\ \text { JQ234673 } & \text { CTCCGCCTGT TTATTTACTT TCACATACAA TTGCCGAACC CCACGACACT AAAGTGTTGG TAAACCAACA CGACTGCCTA TCATGGCTTG ATTTGCAGCC }\end{array}$

AB198665 TAGTAAAAGC GTGATTTTCC TTTGTTTCGG AAGAAGAGGA GCGTTCTCAG CACAACAGTT GAAAGAAATT GCGATAGGGT TGGAGAAGAG TGGATGTCGA BF650015 TAGTAAAAGC GTGATTTTCC TTTGTTTCG AAGAAGAGGA GCGTTCTCAG CACAACAGTT GAAAGAAATT GCCATAGGGT TGGAGAAGAG TGGATGTCGA JQ234673 TAGTAAAAGC GTGATTTTCC TTTGTTTCGG AAGAAGAGGA GCGTTCTCAG CACAACAGTT GAAAGAAATT GCCATAGGGT TGGAGAAGAG TGGATGTCGA

AB198665 TTTCTTTGGT TGGCCCGCAT TTCACCGGAG ATGGACTTAA ATGCGCTTCT GCCGGAGGGT TTTCTATCGA GAACTAAAGG AGTAGGGTTT GTGACAAACA EF650015 TTTCTTTGGT TGGCCCGCAT TTCACCGGAG ATGGACTTAA ATGCGCTTCT GCCGGAGGGT TTTTTATCGA GAACTAAAGG AGTAGGGTTT GTGACAAACA JQ234673 TTTCTTTGGT TGGCCCGCAT TTCACCGGAG ATGGACTTAA ATGCGCTTCT GCCGGAGGGT TTTTTATCGA GAACTAAAGG AGTAGGGTTT GTGACAAACA

AB198665 CATGGGTGCC GCAAAAAGAG GTGTTGAGTC ATGATGCAGT GGGGGGGTTT GTGACTCATT GCGGGTGGAG TTCGGTTCTT GAAGCGCTGT CGTTCGGTGT EF650015 CATGGGTGCC GCAGAAAGAG GTGTTGAGTC ATGATGCAGC GGGGGGTTT GTGACTCATT GTGGGTGGAA TTCTGTTCTT GAAGCGCTGT CGTTCGGTG JQ234673 CATGGGTGCC GCAGAAAGAG GTGTTGAGTC ATGATGCAGC GGGGGGTTT GTGACTCATT GTGGGTGGAA TTCTGTTCTT GAAGCGCTGT CGTTCGGTGT

AB198665 CCCGATGATT GGTTGGCCGT TGTACGCAGA GCAGAGGATC AATAGGGTGT TCATGGTGGA GGAAATAAAG GTGGCGCTGC CATTGGATGA GGAAGATGGA BF650015 CCCGATGATT GGTTGGCCGT TGTACGCAGA GCAGAGGATC AATAGGGTGT TCATGGTGGA GGAAATAAAG GTGGCACTGC CATTGGATGA GGAAGATGGA JQ234673 CCCGATGATT GGTTGGCCGT TGTACGCAGA GCAGAGGATC AATAGGGTGT TCATGGTGGA GGAAATAAAG GTGGCACTGC CATTGGATGA GGAAGATGGA

AB198665 TTTGTGACGG CGATGGAGTT GGAGAAGCGC GTCAGGGAGT TGATGGAGTC GGTAAAGGGG AAAGAAGTGA AGCGCCGTGT GGCGGAATTG AAAATCTCTA BF650015 TTTGTGACGG CGATGGAGTT GGAGAAGCGC GTCAGGGAGT TGATGGAGTC GGTAAAGGGG AAAGAAGTGA AGCGCCGTGT GGCGGAATTG AAAATCTCTA JQ234673 TTTGTGACGG CGATGGAGTT GGAGAAGCGC GTCAGGGAGT TGATGGAGTC GGTAAAGGGG AAAGAAGTGA AGCGCCGTGT GGCGGAATTG AAAATCTCTA

AB198665 CAAAGGCAGC CGTGAGTAAA GGTGGATCGT CCTTGGCTTC TTTGGAGAAG TTCATCAACT CGGTCACTCG TTAA

BF650015 CAAAGGCAGC CGTGAGTAAA GGTGGATCGT CCTTGGTTCC TTTGGAGAAG TTCATCAACT CGGTCACTCG TTA

J2334673 CAAAGCAGC CGTGAGTAAA GGTGGATCGT CCTTGGTTGC TTTGGAGAAG TTCATCAACT CGGTCACTCG TTA

(a)

AB198665 MGEEYKKTHT IVFHTSEEHL NSSIALAKFI TKHHSSISIT IISTAPAESS EVAKIINNPS ITYRGLTAVA LPENLTSNIN KNPVELFFEI PRLQNANLRB BF650015 MGEEYKKTHT IVFHTSEBHL NSSIALAKF I TKHHSSISIT IISTAPAESS EVAKIINNPS ITYRGLTAVA LPENLTSNIN KNPVELFFEI PRLQNANLGB JQ234673 MGEEYKKTHT IVFHTSBEHL NSSIALAKFI TKHHSSISIT IISTAPAESS BVAKIINNPS ITYRGLTAVA LPENLTSNIN KNPVELFFEI PRLQNANLGB

AB198665 ALLDISRKSD IKALIIDFFC NAAFEVSTSM NIPTYFDVSG GAFLLCTFLH HPTLHQTVRG DIADLNDSVE MPGFPLIHSS DLPMSLFYRK TNVYKHFLDT BF 650015 ALLDISRKSD IKALIIDFFC NAAFEVSTSM NIPTYFDVSG GAFLLCTFLH HPTLHQTVRG DIADLNDSVE MPGFPLIHSS DLPMSLFYRK SNVYKHFLD JQ234673 ALLDISRKSD IKALIIDFFC NAAFEVSTSM NIPTYFDVSG GAFLLCTFLH HPTLHQTVRG DIADLNDSVE MPGFPLIHSS DLPMSLFYRK SNVYKHFLDT

AB198665 SLNMRKSSGI LVNTFVALBE RAKBALSNGL YGPTPPLYLL SHTIABPHDT KVLVNQHECL SWLDLQPSKS VIFLCFGRRG AFSAQQLKEI AIGLBKSGCR BF650015 SLNMRKSSGI LVNTFVALEF RAKEALSNGL YGPTPPVYLL SHTIAEPHDT KVLVNQHDCL SWLDLQPSKS VIFLCFGRRG AFSAQQLKEI AIGLEKSGCR JQ234673 SLNMRKSSGI LVNTFVALEF RAKEALSNGL YGPTPPVYLL SHTIAEPHDT KVLVNQHDCL SWLDLQPSKS VIFLCEGRRG AFSAQQLKEI AIGLBKSGCR

AB198665 FLWLARISPE MDLNALLPBG FLSRTKGVGE VTNTWVPQKE VLSHDAVGGF VTHCGWSSVL EALSFGVPMI GWPLYAEQRI NRVFMVEEIK VALPLDEEDG BE650015 FLWLARISPE MDLNALLPEG FLSRTKGVGE VTNTWVPQKE VLSHDAAGGF VTHCGWNSVL EALSFGVPMI GWPLYAEQRI NRVFMVBEIK VALPLDEEDG JQ234673 FLWLARISPE MDLNALLPEG FLSRTKGVGF VTNTWVPOKE VLSHDAAGGE VTHCGWISVL EALSFGVPMI GWPLYABORI NRVFMVEEIK VALPLDEEDG

AB198665 FVTAMELEKR VRELMESVKG KEVKRRVAEL KISTKAAVSK GGSSLASLEK FINSVTR

BF 650015 FVTAMBLEKR VRELMESVKG KBVKRRVAEL KISTKAAVSK GGSSLVALEK FINSVTR

JQ234673 FVTAMELEKR VRELMESVKG KBVKRRVAEL KISTKAAVSK GGSSLVALEK FINSVTR

(b)

Figure 2. Nucleotide sequence (panel (a)) and deduced amino acid sequence (panel (b)) of snapdragon cDNA encoding chalcone 4'-O-glucosyltransferase (4'CGT). Accession number AB198665 is the published Am4'CGT sequence from Antirrhinum majus cv. Yellow Butterfly [11], EF650015 is our cDNA sequence from A.majus cv. Ribbon Yellow (SRY4'CGT), and JQ234673 is our genomic sequence. Nucleotides or amino acids that differed from the published sequence [11] are indicated by highlights.

transforming the vector pSRY4'CGT and using $10 \mathrm{mg} / \mathrm{l}$ hygromycin as selection agent (Table 1); and 13 putative transgenic plants were obtained from a total of 205 cotyledon explants by transforming the vector pSRYAS1 and using $100 \mathrm{mg} / \mathrm{l}$ kanamycin as selection agent (Table 2). Transformation efficiency for SRY'4CGT and SRYAS1 transgenes was $4.8 \%$ and $6.3 \%$, respectively. All transgenic plants were grown in a greenhouse until 


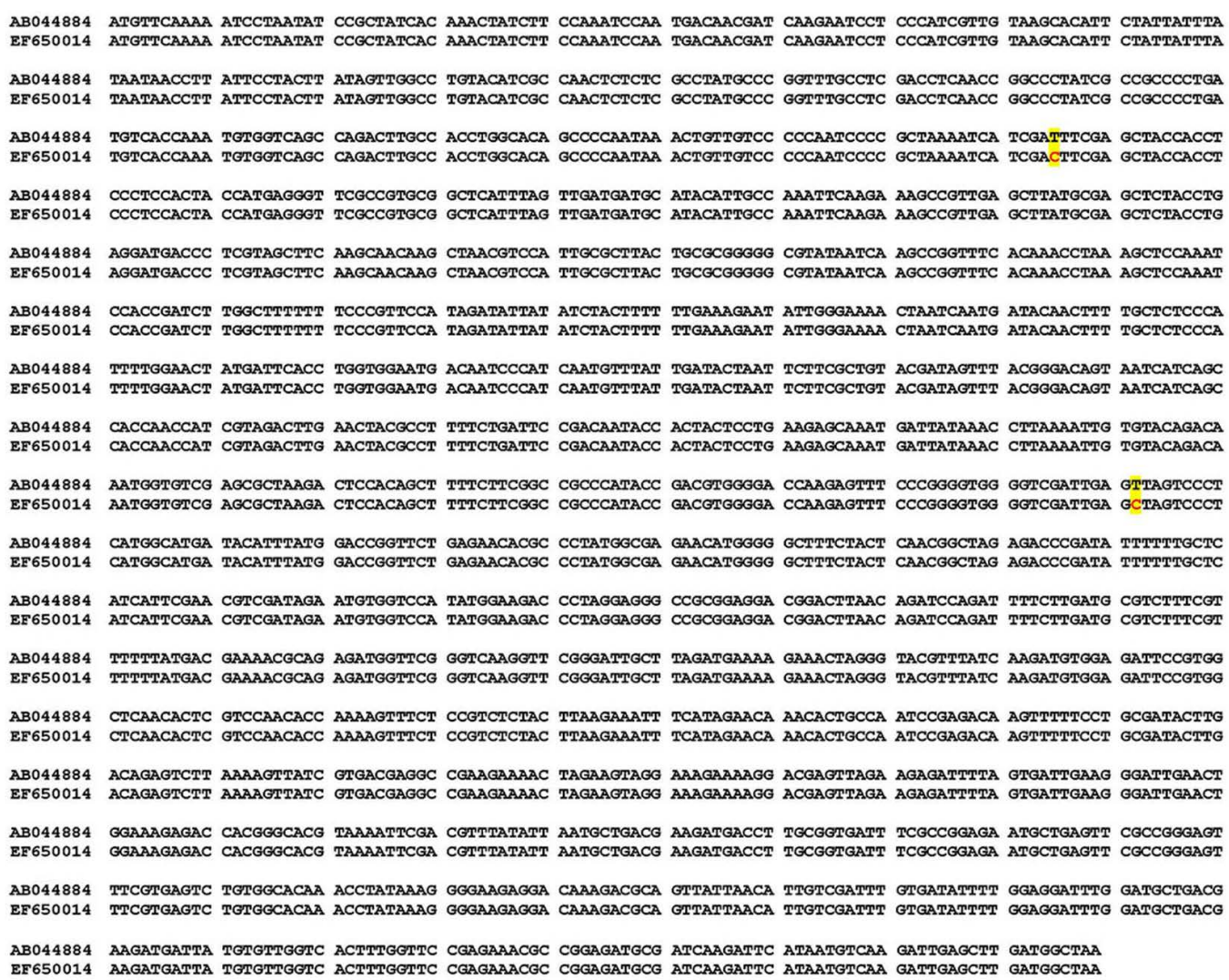

(a)

AB044884 MFKNPNIRYH KLSSKSNDND QESSHRCKHI LLFIITLFLI IVGLYIANSI AYAREASTST GPIAAPDVTK CGQPDLPPGT APINCCPPIP AKIIDFELPP EF650014 MFKNPNIRYH KLSSKSNDND QESSHRCKHI LLFIITLFLI IVGLYIANSL AYARFASTST GPIAAPDVIT CGQPDLPPGT APINCCPPIP AKIIDFELPP

AB044884 PSTIMRVRRA AHLVDDAYIA KFKKAVELMR ALPEDDPRSF KQQANVHCAY CAGAYNQAGF TNLKLQIHRS WLFFPFHRYY IYFFERILGK LINDTTPALP EF650014 PSTTMRVRRA AHUVDDAYIA KFKKAVETMR ALPEDDPRSF KQQANVHCAY CAGAYNQAGF TNLKLQIKRS WLFFPTHRYY IYFFERILGK LINDTTEALP

AB044884 FWNYDSPGGM TIPSMFIDTN SSLYDSLRDS NHQPPTIVDL NYAFSDSDNT TTPEEQMIIN IKIVYRQMVS SAKTPQLFFG RPYRRGDQEF PGVGSIELVP EF650014 FWNYDSPGGM TIPSMPIDTN SSLYDSLRDS NHQPPTIVDL NYAFSDSDNT TTPEEQMIIN LKIVYRQMVS SAKTPQLFFG RPYRRGDQEF PGVGSIRLVP

AB044884 HGMIHLWTGS ENTPYGENMG AFYSTARDPI FFAHHSNVDR MWSINKTLGG PRRTDLTDPD FLDASFVFYD ENAEMVRVKV RDCLDEKKLG YVYQDVEIPV EF650014 HGMIHLWTGS ENTPYGENMG AFYSTARDPI FFAHHSNVDR MWSIWKTLGG PRRTDLTDPD FLDASFVFYD ENAEMVRVKV RDCLDEKKCG YVYQDVEIPW

AB044884 LNTRPTPKVS PSLLKKFHRT NTANPRQVFP AILDRVLKVI VTRPKKTRSR KEKDELEEIL VIEGIELERD HGHVKFDVYI NADEDDLAVI SPENAEFAGS EF650014 INTRPTPKVS PSTLKKFHRT NTAANPROVFP AIIDRVLKYI VTRPKKTRSR KEKDELEEII VIEGIEIERD HGHVKFDVYI NADEDDLAVI SPENAAEFAGS

AB044884 FVSIWHKPIK GKRTKTQILT LSICDILEDI DADEDDYVLV TLVPRNAGDA IKIHNVKIEI DG
EF650014 FVSLWHKPIK GKRTKTOILT LSICDILEDL DADEDDYVLV TLVPRNAGDA IKIHNVKIEI DG

(b)

Figure 3. Nucleotide sequence (panel (a)) and deduced amino acid sequence (panel (b)) of snapdragon cDNA encoding aureusidin synthase (AS1). Accession number AB044884 is the published AmAS1 sequence from Antirrhinum majus cv. Yellow Butterfly [10], EF650014 is our cDNA sequence from A.majus cv. Ribbon Yellow (SRYAS1). Nucleotides that differed from the published sequence [10] are indicated by highlights.

maturity.

Of the 9 transgenic Petunia plants carrying the SRY4'CGT transgene, only a few flowers (from transformants SRY4'CGT-1, -6, -7, -10) showed variegated blue-white sectors (Table 1). Those transformants that produced variegated blue-white flowers, at the late stage of flowering, more and more new-born flowers produced variegated blue-white flowers, and the areas of white sectors increased in the blue background of the petals. As time passed, most of the old flowers without alteration in pigmentation faded, and the number of new-born flowers carrying more white sectors/regions in the background blue color increased. Finally, petal color was changed to completely white in all the late-born flowers (Figure 5). In contrast, of the 13 transgenic Petunia plants carrying SRYAS1 transgene, three (SRYAS1-4, -6, -12) showed variegated blue-white flowers at the late stage of flowering (Table 2): a few variegated flowers carrying small portions of white sectors in the background blue petals were observed in these 3 transgenic lines. However, complete white flowers were not observed. The growth 


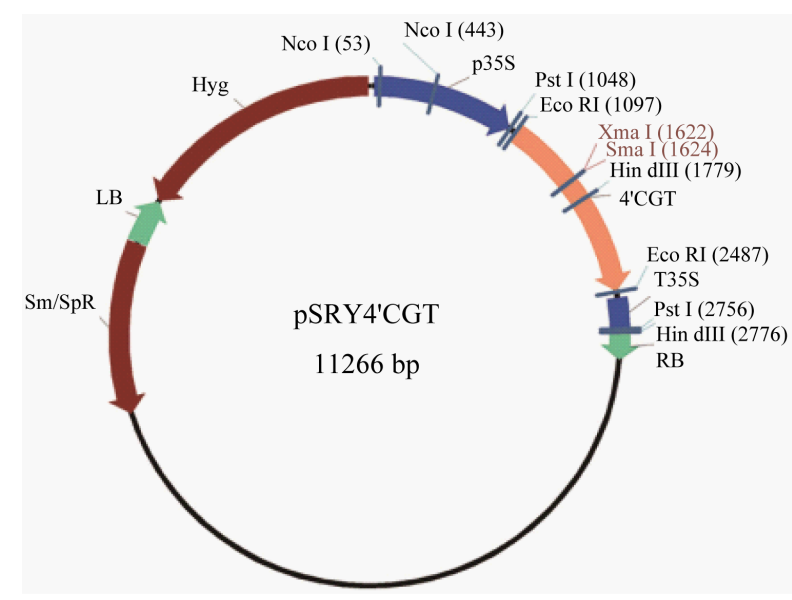

(a)

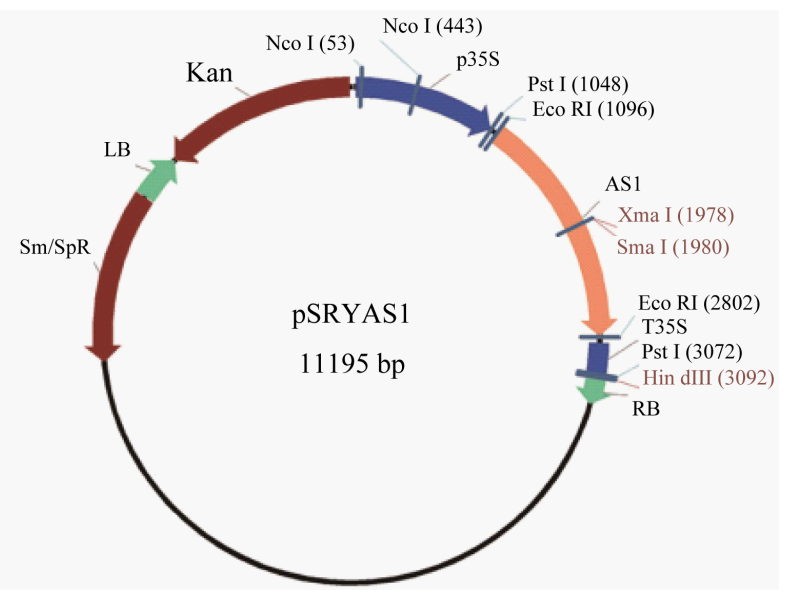

(b)

Figure 4. Restriction maps of transformation vectors pSRY4'CGT (a) and pSRYAS1 (b).

Table 1. Alteration in flower color and inheritance in $\mathrm{T}_{1}$ progeny in transgenic Petunia plants carrying SRY4'CGT transgene.

\begin{tabular}{|c|c|c|c|c|c|}
\hline \multirow{2}{*}{ Transgenic plant } & \multirow{2}{*}{ Flower color } & \multicolumn{2}{|c|}{$\begin{array}{l}\text { No. of resistant }(\mathrm{R}) \text { and sensitive }(\mathrm{S}) \\
\text { seedlings from } \mathrm{T}_{0} \text { selfed seeds }\end{array}$} & \multirow{2}{*}{ Test ratio (R:S) } & \multirow{2}{*}{ Remarks } \\
\hline & & $\mathrm{R}$ & S & & \\
\hline SRY4'CGT-1 & Blue-white & & & & No seeds \\
\hline SRY4'CGT-2 & Blue & 178 & 172 & $1: 1$ & \\
\hline SRY4'CGT-3 & Blue & & & & No seeds \\
\hline SRY4'CGT-4 & Blue & 282 & 170 & $2: 1$ & \\
\hline SRY4'CGT-5 & Blue & & & & No seeds \\
\hline SRY4'CGT-6 & Blue-white & 265 & 308 & $1: 1$ & \\
\hline SRY4'CGT-7 & Blue-white & 577 & 191 & $3: 1$ & \\
\hline SRY4'CGT-10 & Blue-white & 138 & 43 & $3: 1$ & \\
\hline SRY4'CGT-12 & Blue & 189 & 98 & $2: 1$ & \\
\hline
\end{tabular}

Seeds were germinated on MS medium containing $10 \mathrm{mg} / \mathrm{l}$ hygromycin for 3 weeks.

pattern of a typical transgenic SRYAS1 plant carrying variegated flowers is shown in Figure 6. In addition, the amount and appearance of variegated blue-white flowers during the late stage of flowering (or plant senescence) in transgenic SRYAS1 plants were less than and later than, respectively, those of transgenic SRY4'CGT plants.

\subsection{Gene Expression in $\mathrm{T}_{0}$ Transgenic SRYAS1 and SRY4'CGT Plants Carrying Variegated Blue-White Flowers}

To examine the presence and expression of transgenes in transgenic plants, total RNA was isolated from variegated blue-white flowers in three transgenic SRYAS1 plants (SRYAS1-4, -6, -12) and four transgenic SRY4'CGT plants (SRY4'CGT-1, -6, -7, -10). In addition, total RNA was also isolated from blue flowers in wild-type $P$. hybrida. RT-PCR analysis was carried out to investigate the expression of transgene in transgenic plants (Figure 7). As expected, no transcripts were detected in wild-type Petunia flowers when gene-specific SRYAS1 or SRY4'CGT primers were employed. Transgenic SRYAS1 transcripts were detected in all three transgenic SRYAS1 plants, and transgenic SRY4'CGT transcripts were detected in all four transgenic SRY4'CGT plants (Figure 7). Thus, these results demonstrate clearly the presence and expression of transgenes in transgenic plants we examined. 

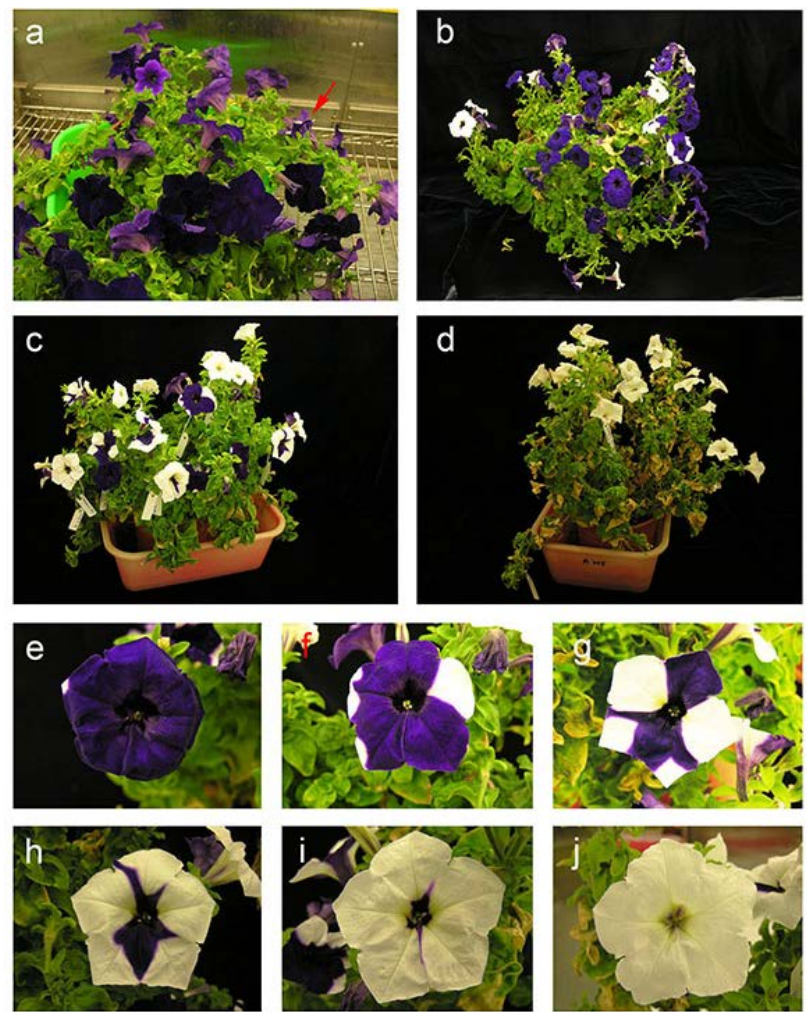

Figure 5. Alternation of flower coloration in transgenic Petunia plants carrying SRY4'CGT transgene. (a) At the beginning of flowering, only a few flowers (indicated by arrow) showed white sectors at the edge of the blue petals. (b) and (c) Over time, flowers with regular blue color faded, and at the middle and late stages of flowering variegated flowers carrying white sectors in a blue background, or complete white flowers, appeared. (d) During the late stage of flowering, variegated blue-white flowers gradually faded and new flowers emerging at that stage were almost or all white in color. As a result, all the flowers at the late-flowering stage in transgenic plants looked white in color. (e) to (j) Flower phenotypes from a spot of white color in the blue background to complete white color observed in the same transgenic plant.

Table 2. Alteration in flower color and inheritance in $\mathrm{T}_{1}$ progeny in transgenic Petunia plants carrying SRYAS1 transgene.

\begin{tabular}{|c|c|c|c|c|c|}
\hline \multirow{2}{*}{ Transgenic plant } & \multirow{2}{*}{ Flower color } & \multicolumn{2}{|c|}{$\begin{array}{l}\text { No. of resistant }(\mathrm{R}) \text { and sensitive }(\mathrm{S}) \\
\text { seedlings from } \mathrm{T}_{0} \text { selfed seeds }\end{array}$} & \multirow{2}{*}{ Test ratio (R:S) } & \multirow{2}{*}{ Remarks } \\
\hline & & $\mathrm{R}$ & S & & \\
\hline SRYAS1-1 & Blue & 123 & 121 & $1: 1$ & \\
\hline SRYAS1-2 & Blue & 55 & 16 & $3: 1$ & \\
\hline SRYAS1-3 & Blue & 437 & 188 & $2: 1$ & \\
\hline SRYAS1-4 & Blue-white & 150 & 56 & $3: 1$ & \\
\hline SRYAS1-5 & Blue & 196 & 85 & $2: 1$ & \\
\hline SRYAS1-6 & Blue-white & 134 & 59 & $2: 1$ & \\
\hline SRYAS1-7 & Blue & 0 & 340 & & \\
\hline SRYAS1-8 & Blue & & & & No seeds \\
\hline SRYAS1-9 & Blue & 110 & 109 & $1: 1$ & \\
\hline SRYAS1-10 & Blue & 394 & 181 & $2: 1$ & \\
\hline SRYAS1-11 & Blue & 0 & 466 & & \\
\hline SRYAS1-12 & Blue-white & 299 & 110 & $3: 1$ & \\
\hline SRYAS1-13 & Blue & & & & No seeds \\
\hline
\end{tabular}

Seeds were germinated on MS medium containing $100 \mathrm{mg} / \mathrm{l}$ kanamycin for 3 weeks. 

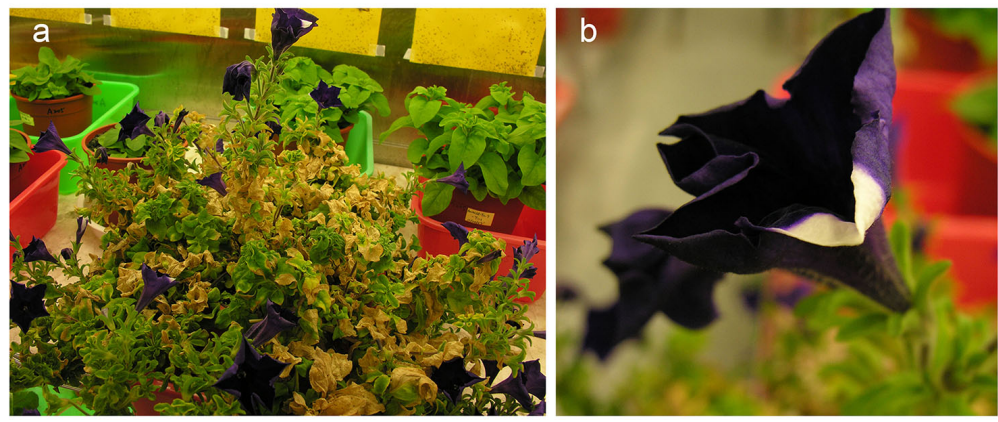

Figure 6. Flower phenotype in transgenic Petunia plants carrying SRYAS1 transgene. (a) Most transgenic flowers were blue in color, similar to wild-type flowers. (b) A small number of transgenic flowers had white sectors on the edge of a blue petal.

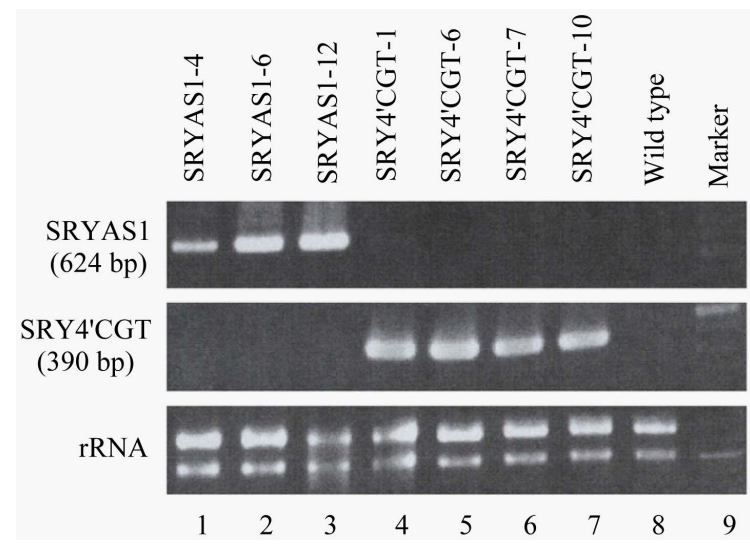

Figure 7. Gene expression in $\mathrm{T}_{0}$ transgenic SRYAS1 and SRY4'CGT plants carrying variegated blue-white flowers. Total RNA was isolated from variegated blue-white flowers in three transgenic SRYAS1 plants (SRYAS1-4, -6, -12) and four transgenic SRY4'CGT plants (SRY4'CGT-1, -6, -7, -10). "Wild type" represented RNA sample isolated from wild-type Petunia flowers (blue color). RT-PCR was conducted for 25 cycles using F1 and R2 for amplification of SRYAS1 gene (expected size of this fragment was $624 \mathrm{bp}$ ), and F2 and R3 primers for amplification of SRY4'CGT gene (expected size of this fragment was $390 \mathrm{bp}$ ). The rRNA visualized by ethidium bromide staining is indicated as a loading control.

\subsection{Inheritance in $\mathrm{T}_{1}$ Progeny of Transgenic Plants}

The stable integration of the T-DNA region in two transformation vectors (Figure 4) into the petunia chromosomal genome was investigated by germinating $T_{1}$ seeds from self-fertilized $T_{0}$ transgenic plants on MS medium containing $10 \mathrm{mg} / \mathrm{l}$ hygromycin (Table 1 ) or $100 \mathrm{mg} / \mathrm{l}$ kanamycin sulfate (Table 2). In transgenic plants carrying SRY4'CGT transgene (Table 1), $\mathrm{T}_{1}$ seeds were not available from three transformants (SRY4'CGT-1, -3, -5). Hygromycin-resistant and hygromycin-sensitive $T_{1}$ seedlings segregated in a 3:1 ratio in 2 transformants (SRY4'CGT-7 and SRY4'CGT-10), indicating the presence of single copy of the hygromycin-resistant transgene in the nuclear genomes of the 2 lines (Table 1). Since the transformation vector pSRY4'CGT contains expression cassettes for the selection marker gene (hygromycin resistant gene) and snapdragon SRY4'CGT transgene within the T-DNA region (Figure 4(a)), it is reasonable to predict that the same copy number of the hygromycin resistant transgene and SRY4'CGT transgene will be found in each transformant.

All transgenic plants carrying SRYAS1 transgene are shown in Table 2. No seeds were obtained from two transformants, SRYAS1-8 and SRYAS1-13. All $\mathrm{T}_{1}$ seedlings from the two transformants SRYAS1-7 and SRYAS1-11 were sensitive to the selection agent kanamycin, suggesting that these two lines may not contain the foreign T-DNA fragment and can be considered to be false-positive clones. Thus, transformation efficiency in transgenic SRYAS1 plants was recalculated as $5.4 \%$. Single insertion of the nptII transgene was determined in 
three transgenic plants SRYAS1-2, SRYAS1-4 and SRYAS1-12, as the segregation ratio of resistant and sensitive seedlings in these 3 transgenic plants was 3:1 (Table 2). Similarly, as transformation vector pSRYAS1 contains expression cassettes for the selection marker gene (nptII) and snapdragon SRYAS1 transgene within the T-DNA region (Figure $4(b)$ ), it is reasonable to predict the same copy number of the nptII transgene and SRYAS1 transgene will be found in each transformant.

\subsection{Gene Expression in $\mathrm{T}_{1}$ Transgenic SRY4'CGT Plants}

Five $\mathrm{T}_{1}$ hygromycin-resistant seedlings were randomly selected in hygromycin-containing medium from $\mathrm{T}_{0}$ selfed seeds of transgenic plant SRY4'CGT-7 that showed significant alteration in flower coloration, and grown in a greenhouse until maturity. Instead of no phenotypic change in $3 \mathrm{~T}_{1}$ transgenic plants, obvious variegated blue-white flowers were still observed in $2 \mathrm{~T}_{1}$ transgenic plants (Figure 8). However, no complete white flowers
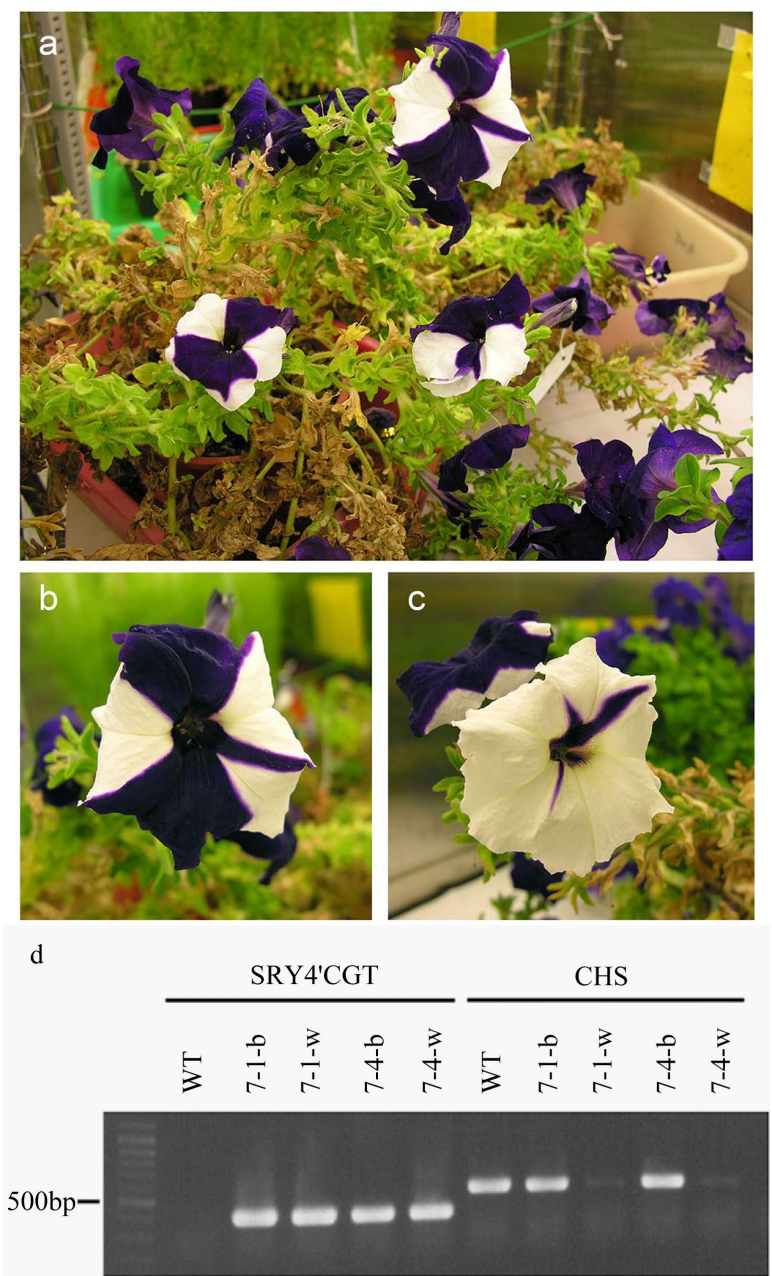

Figure 8. Flower phenotype and gene expression patterns in $\mathrm{T}_{1}$ transgenic SRY4'CGT-7 plants. (a) Mature plant of $\mathrm{T}_{1}$ transgenic SRY4'CGT-7- $\mathrm{T}_{1}-1$. Five hygromycin-resistant $\mathrm{T}_{1}$ seedlings from transgenic SRY4'CGT-7 $\mathrm{T}_{0}$ plant were randomly-selected and grown until maturation. Among them, $2 \mathrm{~T}_{1}$ progenies, designed as SRY4'CGT-7- $\mathrm{T}_{1}-1$ and SRY4'CGT-7- $\mathrm{T}_{1}-4$, showed variegated blue-white flowers as parental SRY4'CGT-7 $\mathrm{T}_{0}$ plant, whereas no alteration in flower color was observed in the rest of the three $\mathrm{T}_{1}$ progenies. (b) An enlarged flower in transgenic SRY4'CGT-7- $\mathrm{T}_{1}-1$ plant. (c) An enlarged flower in transgenic SRY4'CGT-7- $\mathrm{T}_{1}-4$ plant. (d) Gene expression patterns in $\mathrm{T}_{1}$ transgenic SRY4'CGT-7 flowers. RNA was isolated from blue sectors of $\mathrm{T}_{1}$ transgenic flowers of SRY4'CGT-7- $\mathrm{T}_{1}-1$ (abbreviated as 7-1-b) or white sectors of the same plant (abbreviated as 7-1-w), or from blue sectors of $\mathrm{T}_{1}$ transgenic flowers of SRY4'CGT-7- $\mathrm{T}_{1}-4$ (abbreviated as 7-4-b) or white sectors of the same plant (abbreviated as 7-4-w). RT-PCR was conducted for 25 cycles using F2 and R3 primers for amplification of SRY4'CGT gene (expected size of this fragment was $390 \mathrm{bp}$ ), and using C1 and C2 primers for amplification of CHS gene (expected size of this fragment was $558 \mathrm{bp}$ ). 
were found even at the late stage of flowering in any lines. To examine the gene expression in these variegated flowers, white sectors and blue sectors were dissected from variegated flowers in $2 \mathrm{~T}_{1}$ transgenic lines, RNA was isolated, and RT-PCR was performed using gene-specific primers (Figure 8(d)). As predicted, no SRY4'CGT transcripts were detected in wild-type Petunia flowers. Transgenic SRY4'CGT transcripts were detected in both blue and white sectors from these two transgenic lines. When specific primers were employed for an upstream gene chalcone synthase (CHS) in the flavonoid/aurone biosynthetic pathway, $\mathrm{CHS}$ transcripts were only detected in blue sectors but not in white sectors of the transgenic plants examined (Figure 8(d)), suggesting that the expression of CHS gene was suppressed in the white sectors of transgenic plants.

\subsection{High-Performance Liquid Chromatography (HPLC) Profile in $\mathrm{T}_{1}$ Transgenic Flowers}

To examine the pigment profile in transgenic flowers, and more importantly, to investigate whether the appearance of white sectors in blue background petals is due to the loss of anthocyanins, white sectors and blue sectors were dissected from variegated flowers in $\mathrm{T}_{1}$ transgenic SRY4'CGT and SRYAS1 lines, and ground into a powder by liquid nitrogen. After extraction, crude extracts were analyzed by HPLC. For each sample, similar HPLC patterns were obtained when monitoring was performed at $360 \mathrm{~nm}$ (for measurement of chalcones, flavones), $400 \mathrm{~nm}$ (for measurement of aurones) or $520 \mathrm{~nm}$ (for measurement of anthocyanidins). As shown in Figure 9, an absorption peak was detected in all samples when retention time (RT) approached 2.51 to 2.59 min. However, two peaks at RT $=11.57 \mathrm{~min}$ and $\mathrm{RT}=11.78 \mathrm{~min}$ were detected in blue sectors from transgenic SRY4'CGT7-T $\mathrm{T}_{1}-1$ flowers (designed as B1) but not in white sectors from transgenic SRY4'CGT-7-T 1 -1 flowers (designed as W1). Again, two peaks at RT $=11.71 \mathrm{~min}$ and $\mathrm{RT}=11.82 \mathrm{~min}$ were detected in blue sectors from transgenic SRY4'CGT-7-T 1 -4 flowers (designed as B2) but not in white sectors from transgenic SRY4'CGT-7-T -4 flowers (designed as W2). Only one peak at RT = 11.85 min was detected in blue sectors from transgenic SRYAS1-3$\mathrm{T}_{1}-5$ flowers (designed as B3) but not in white sectors from transgenic SRYAS1-3-T -5 flowers (designed as W3); however, the HPLC pattern in B3 was distinguishable from the HPLC patterns in B1 and B2, which represented two individual $\mathrm{T}_{1}$ plants from the same $\mathrm{T}_{0}$ parent SRY4'CGT-7 plant. Besides the constitutive peak at RT $=2.51-2.59 \mathrm{~min}$ in all blue and white samples, almost no significant peaks were detected from white sectors (W1, W2, W3), suggesting that colored pigments may not exist or may accumulate at very low level in these white sectors. HPLC patterns monitored at $360 \mathrm{~nm}, 400 \mathrm{~nm}$ and $520 \mathrm{~nm}$ are shown in Figures 9(a)-(c), respectively.

\subsection{Compound Identification by Tandem Mass Spectrometry (MS/MS)}

To further identify the major compounds in these peaks, tandem mass spectrometry (MS/MS) was employed to identify fragmentation patterns of different anthocyanins. As shown in Figure 10 and Figure 11, several major peaks obtained from different retention times were chosen and then analyzed. The molecular weight of each fragment was measured as mass-to-charge ratio $(\mathrm{m} / \mathrm{z})$ in the ionization process, and the most abundant fragment in each RT was considered as $100 \%$. Compound identification, mainly based on molecular ions and product ions, was performed with the aid of existing literatures spectral analysis regarding flavonoids/anthocyanins [19]-[22]. Cyanidin triglucoside was found as major compound in the RT = $2.62 \mathrm{~min}$ fraction (Figure 10; Figure 11). Fragmentation pattern of cyanidin 3,5,5'-triglucoside is shown in Figure 12(a).

Next, major compounds were identified in peaks around RT = 11.51 - 12.96 min (Figure 10; Figure 11). It is worth noting that these peaks can be detected only in the blue sectors of transgenic flowers but not in white sectors. The red color of cyanidin and its glucoside derivatives (cyanidin glucoside, cyanidin diglucoside, cyanidin malonyldiglucoside), and mauve (pale purple) color of malvidin and its derivative malvidin-3-( $p$-coumaroyl)rutinoside-5-glucoside were identified in these tissues. Fragmentation patterns of these anothcyanins are shown in Figure 12(b), Figure 13(a) and Figure 13(b). These data demonstrated clearly that the major compounds of anthocyanins extracted from the blue sectors of transgenic SRY4'CGT and SRYAS1 flowers are cyanidin, malvidin and their derivatives. In contrast, cyanidin or malvidin was not detected or was accumulated at a very low level in the white sectors of transgenic flowers, as a result, white color was observed.

Finally, to evaluate whether aurones (the target yellow pigment) can be detected in our analysis system, yellow petals from snapdragon were excised, and sample preparation and HPLC-DAD-MS/MS were performed as previously described. As shown in Figure 14(a), several peaks including RT = 2.51 min were detected in HPLC analysis. Compound identification in the fraction at $\mathrm{RT}=11.25 \mathrm{~min}$ revealed that aureusidin glucoside and its 

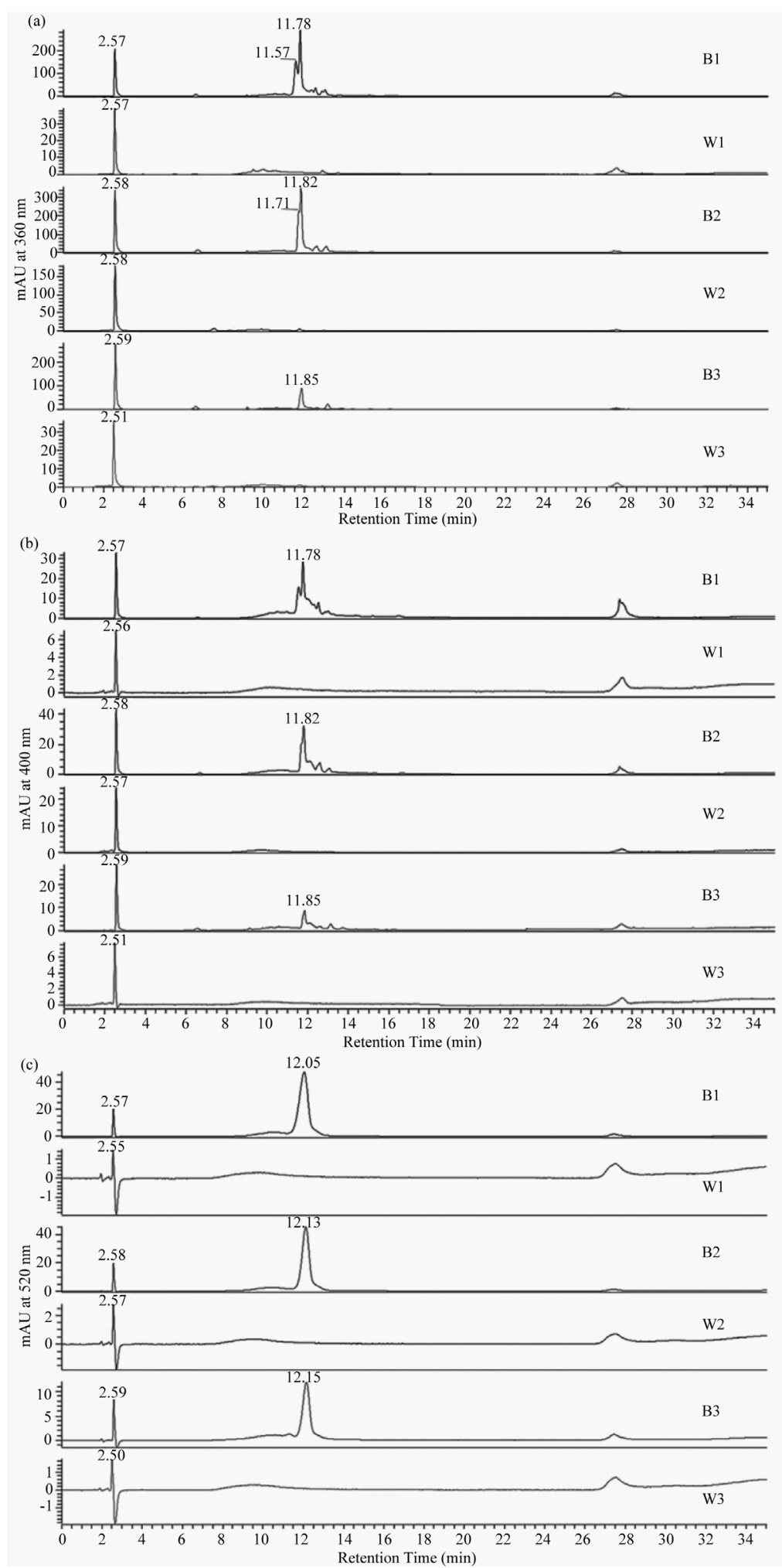

Figure 9. HPLC chromatograms of transgenic Petunia flowers detected at different absorption spectra. (a) $360 \mathrm{~nm}$; (b) $400 \mathrm{~nm}$; (c) $520 \mathrm{~nm}$. Abbreviations: B1, Blue sectors from transgenic SRY4'CGT-7-T $1-1$ flowers; W1, White sectors from transgenic SRY4'CGT-7-T $1-1$ flowers; B2, Blue sectors from transgenic SRY4'CGT-7- $\mathrm{T}_{1}-4$ flowers; W2, white sectors from transgenic SRY4'CGT-7- $\mathrm{T}_{1}-4$ flowers; B3, Blue sectors from transgenic SRYAS1-3-T $T_{1}-5$ flowers; $W 3$, white sectors transgenic SRYAS1-3- $T_{1}-5$ flowers. 


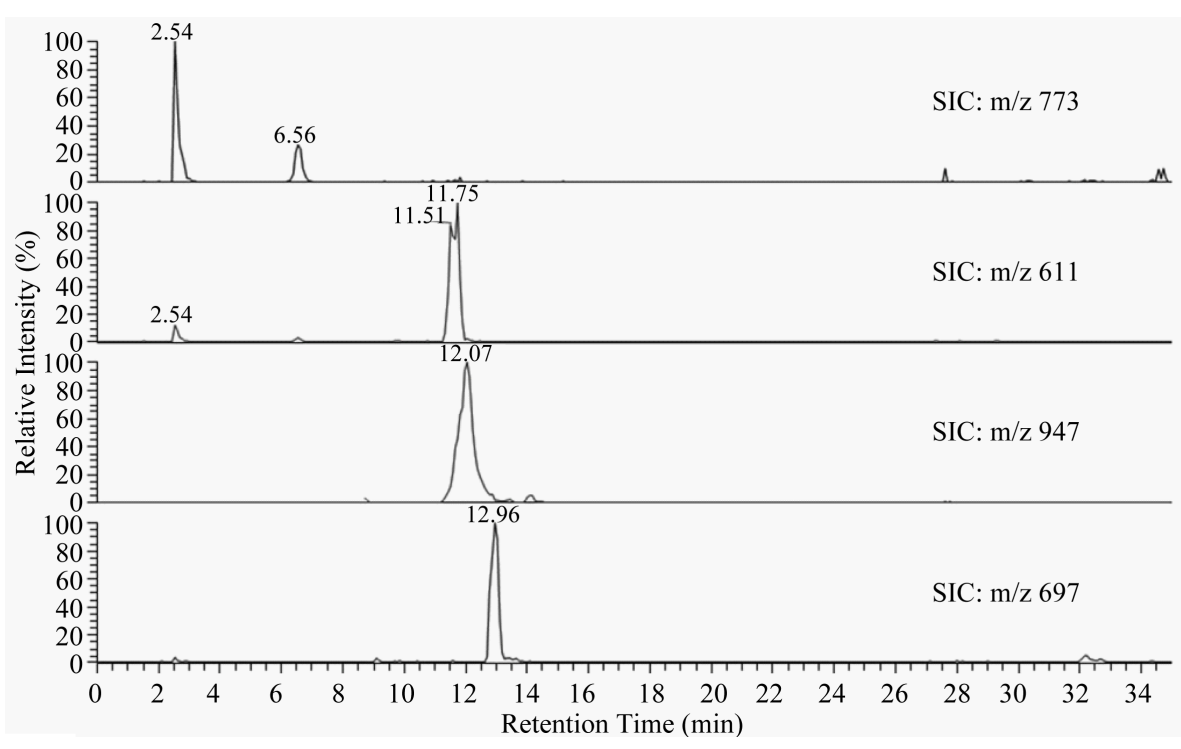

Figure 10. Selected ion chromatograms (SIC) of Electrospray Ionization (ESI) mass spectra detecting precursor ions from cyanidin 3,5,3'-triglucoside $(\mathrm{m} / \mathrm{z} 773)$, cyanidin 3,5-diglucoside $(\mathrm{m} / \mathrm{z}$ 611), malvidin-3-(p-coumaroyl)-rutinoside-5-glucoside $(\mathrm{m} / \mathrm{z} 947)$, and cyanidin malonyldiglucoside $(\mathrm{m} / \mathrm{z}$ 697).

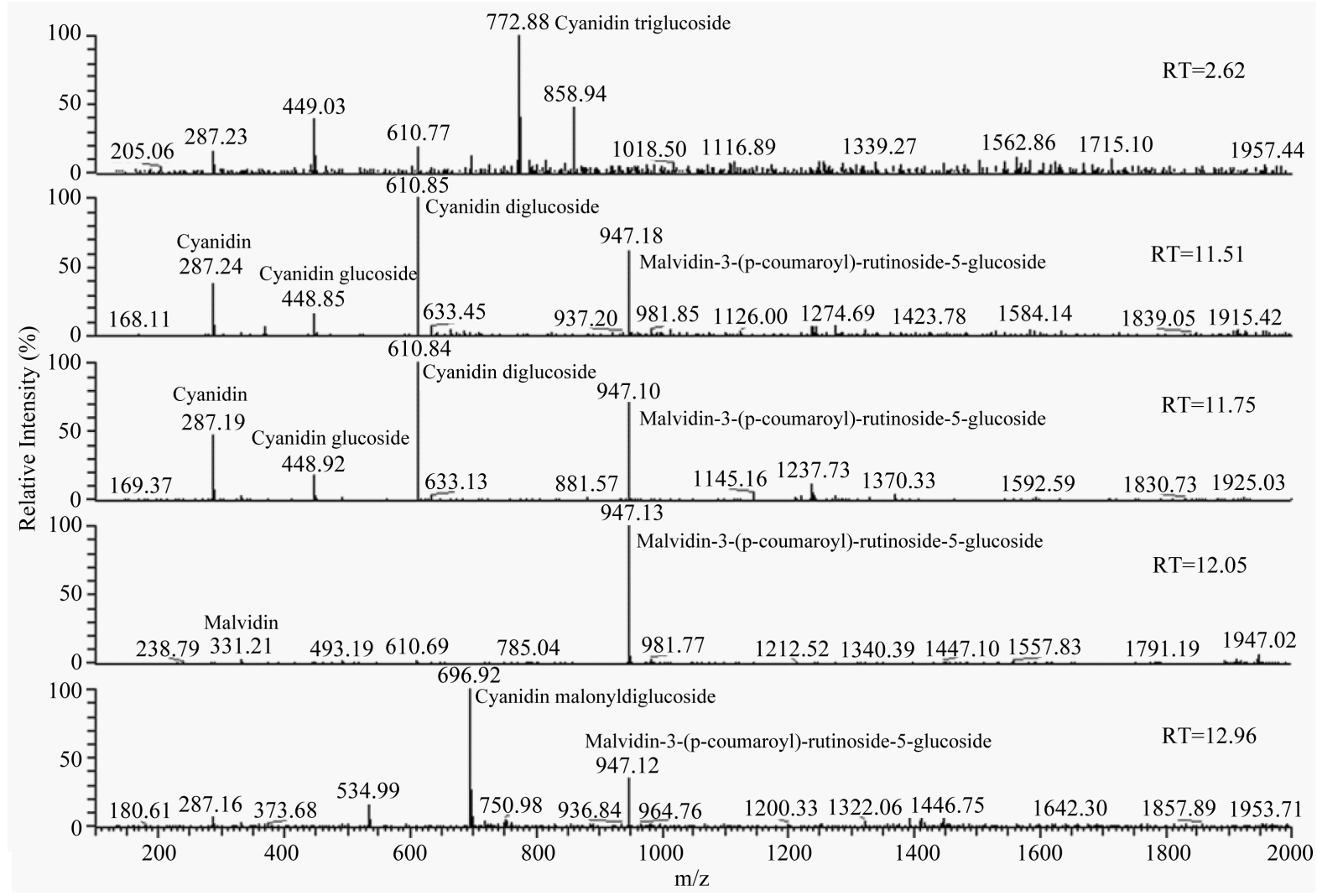

Figure 11. Mass spectra at different retention times.

dimer are the major compounds in that fraction (Figure 14(b)). It is worth pointing out that aureusidin is a yellow pigment belonging to the aurone, and aureusidin 6-O-glucoside (yellow pigment) occurs in the yellow petals of snapdragon. In addition, aureusidin 6-O-glucoside is the product of aureusidin synthase, one of our target 


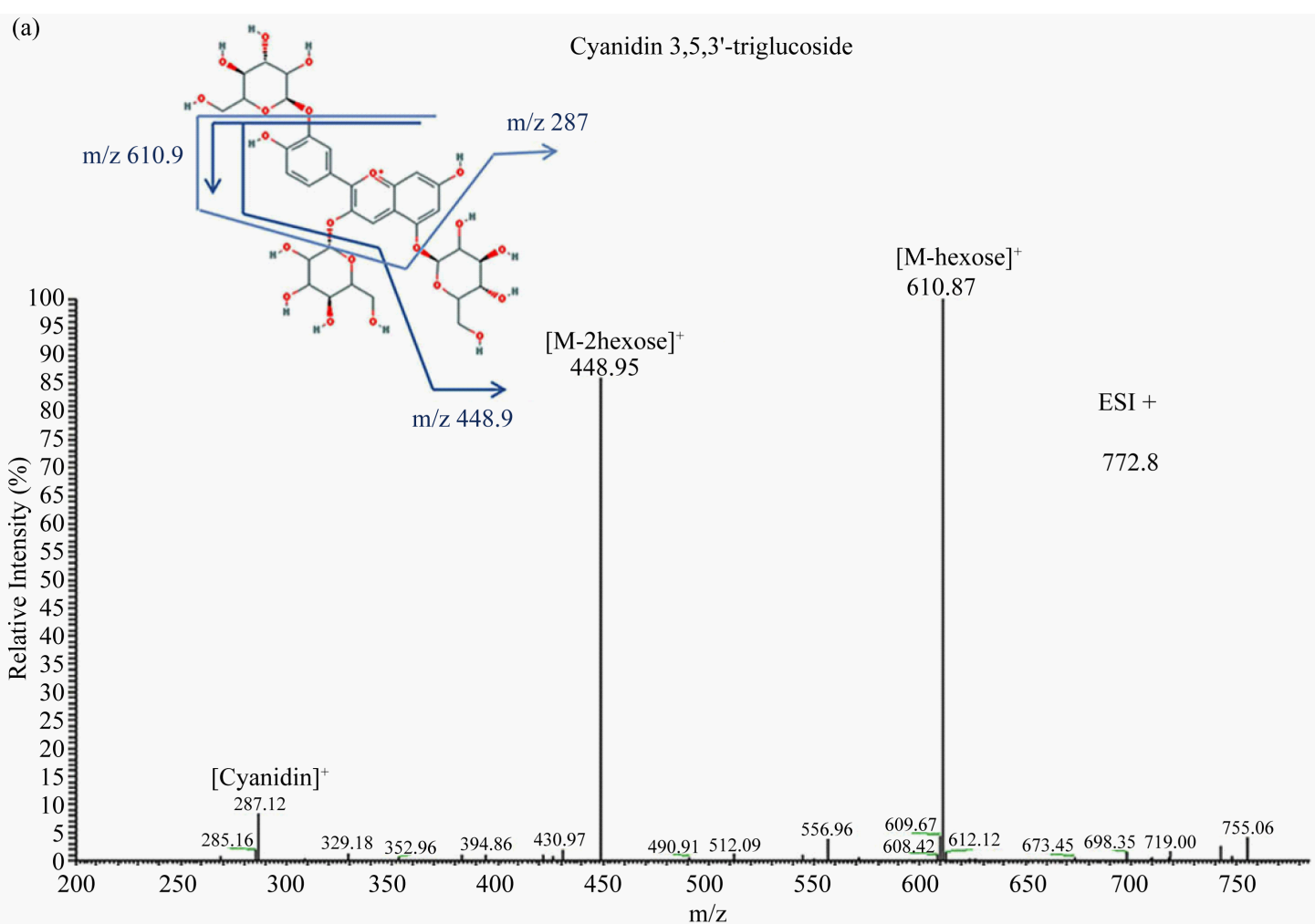

(b)

m/z 448.99

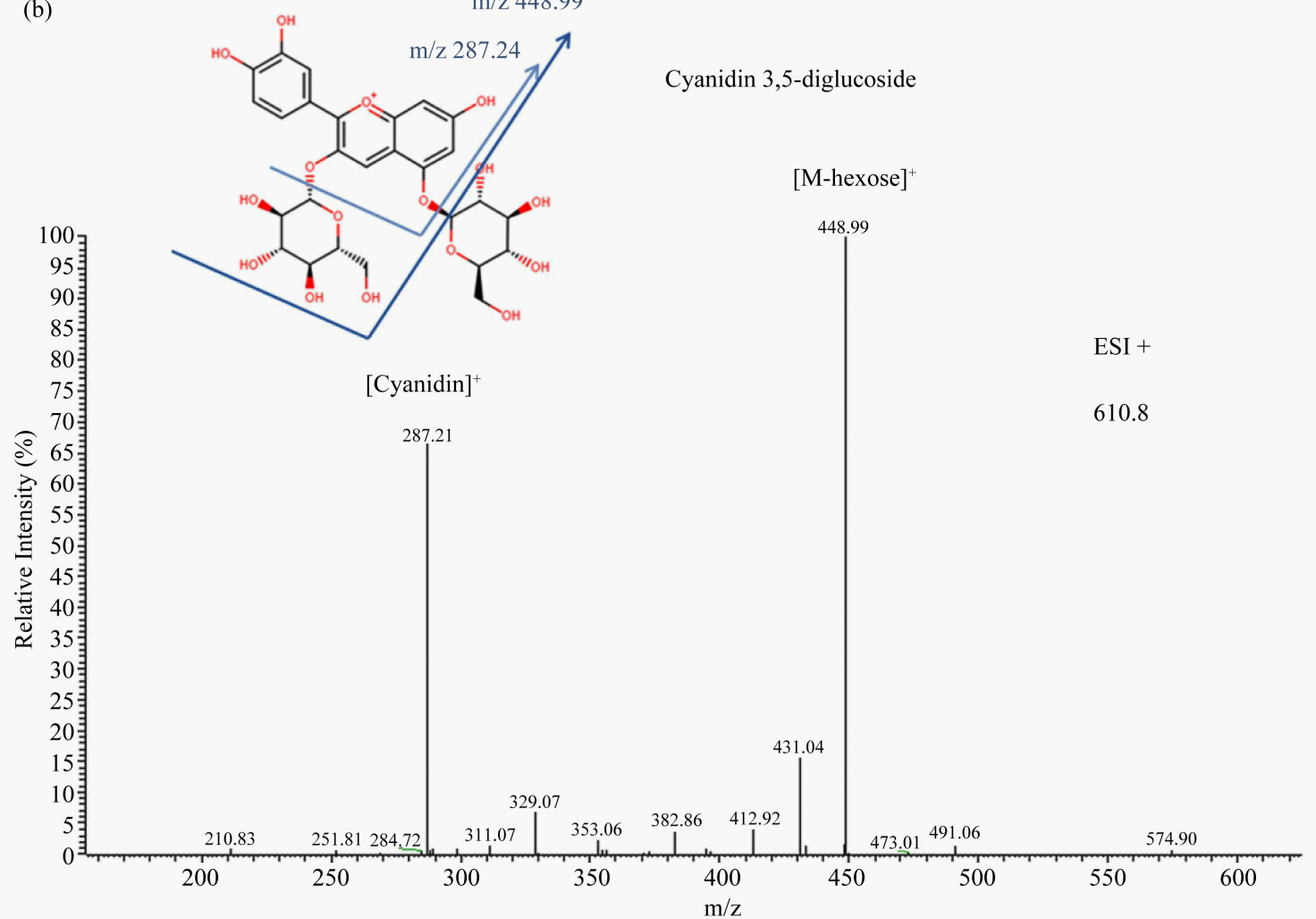

Figure 12. (a) MS/MS production ion spectrum of $m / z 772.8$ ion observed in the MS spectrum for cyanidin 3,5',3'triglucoside. (b) MS/MS production ion spectrum of $\mathrm{m} / \mathrm{z} 610.8$ ion observed in the MS spectrum for cyanidin 3,5diglucoside. 
(a)

Malvidin-3-(p-coumaroyl)-rutinoside-5-glucoside

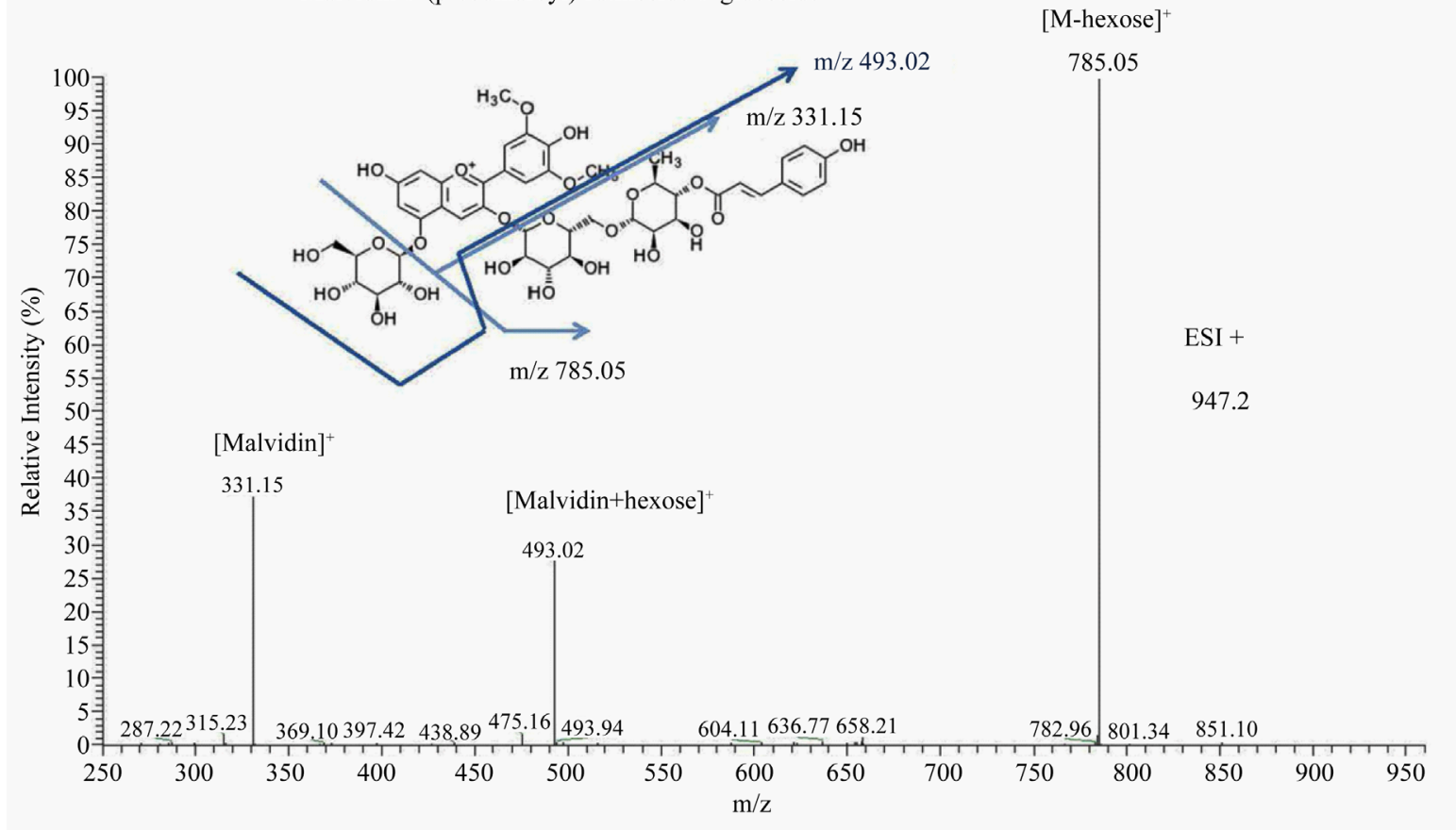

(b)

Cyanidin malonyldiglucoside

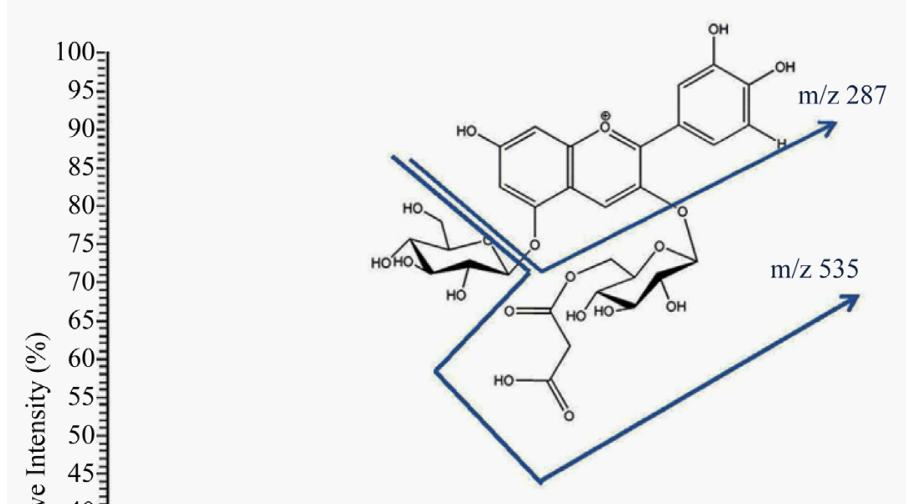

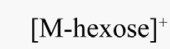

[Cyanidin $^{+}$

䓌 35

yanidin

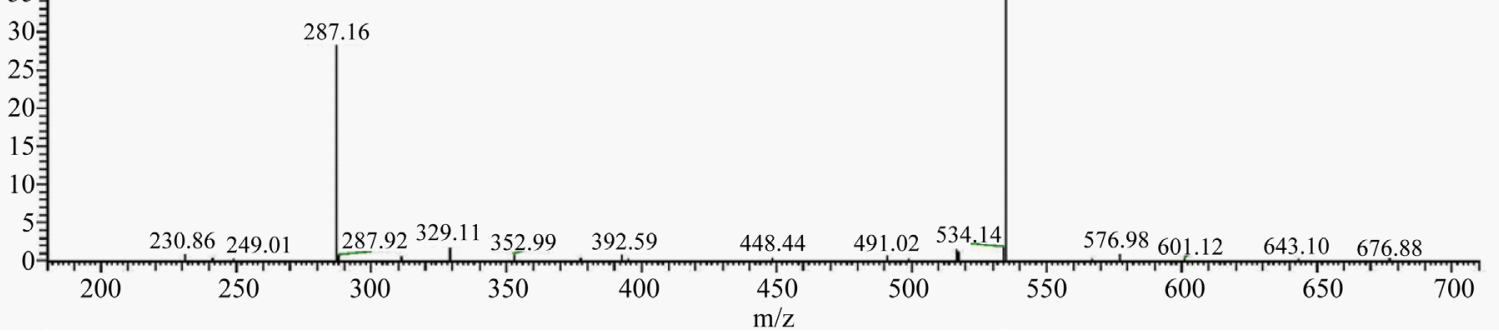

Figure 13. (a) MS/MS production ion spectrum of $m / z 947.2$ ion observed in the MS spectrum for malvidin-3-( $p$-coumaroyl)-rutinoside-5-glucoside. (b) MS/MS production ion spectrum of $\mathrm{m} / \mathrm{z} 699.3$ ion observed in the MS spectrum for cyanidin malonyldiglucoside.

genes (SRYAS1) in this study. The fragmentation pattern aureusidin glucoside is shown in Figure 14(c). In conclusion, yellow pigment of aureusidin glucoside can be detected in our analysis system. However, aureusidin or aureusidin glucoside was not found in our transgenic Petunia flowers. In addition, genistin 7-O-glucuronide (an isoflavone derivative) was identified as a major compound in fraction at RT = 17.89 min (data not shown). 


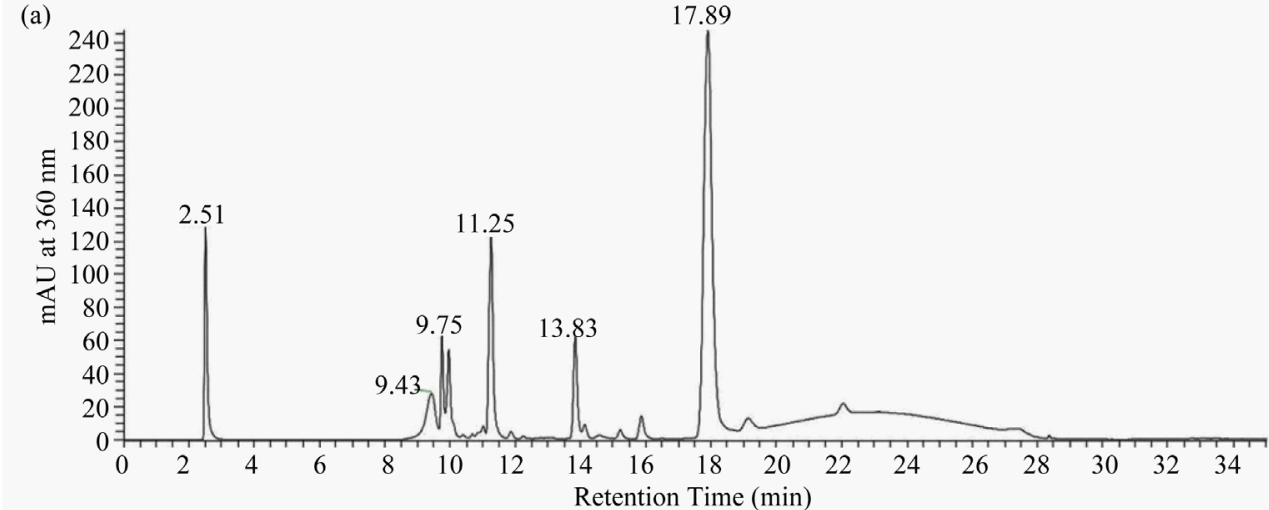

(b) [Aureusidin Glucoside -H]
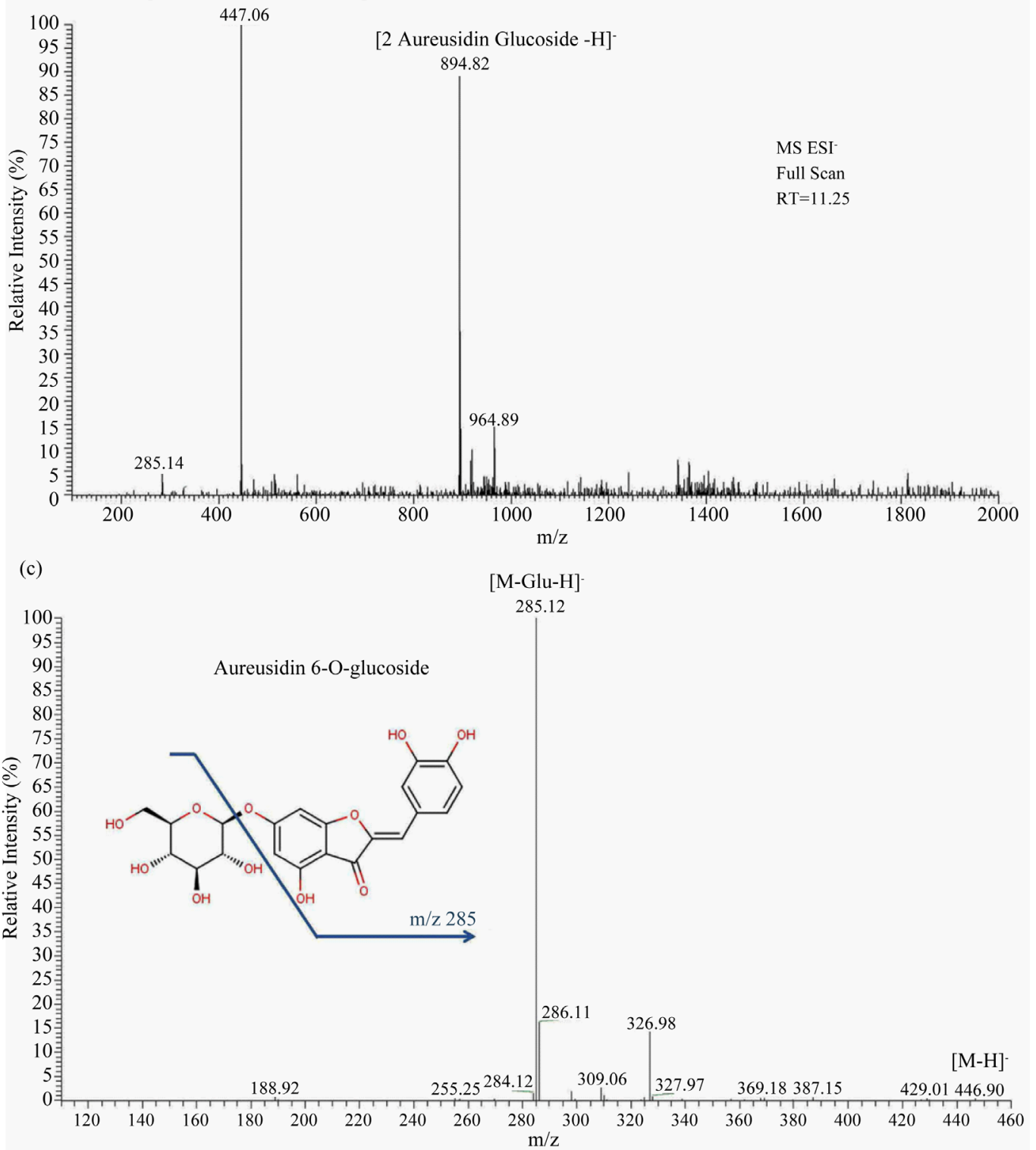

Figure 14. (a) HPLC chromatogram of yellow flowers from snapdragon detected at $360 \mathrm{~nm}$; (b) Aureusidin glycoside and its dimer are the major compounds in the fraction at RT $=11.25 \mathrm{~min}$. (c) MS/MS product ion spectrum of $\mathrm{m} / \mathrm{z} 446.9$ ion observed in the MS spectrum for aureusidin 6-O-glucoside. 
HPLC coupled with tandem mass spectrometry was employed to analyze the blue and white sectors from variegated flowers of transgenic SRY4'CGT and SRYAS1 plants, and the results clearly demonstrated that cyanidin, malvidin, and their derivatives are present in the blue sectors, but none of anthocyanins are present in white sectors, causing the white phenotype.

\section{Discussion}

Flavonoids/anthocyanins are major contributors to flower coloration. As a result, most studies on genetic engineering of flower color have been carried out via the flavonoid biosynthetic pathway [3] [6] [7] [23] [24]. Introduction of an additional CHS gene into different varieties of Petunia hybrida resulted in variegated flowers with white sectors in some transgenic lines and complete white flowers in other transgenic lines [25] [26]. Molecular dissection of these transformants showed that both the introduced and endogenous CHS genes were "turned down" or silenced to different degrees, and as a result, loss of pigmentation in transgenic petals was observed [25] [26]. CHS is a key enzyme catalyzing one molecule of 4-coumaroyl-CoA and three molecules of malonylCoA into one molecule of naringenin chalcone, which is the central intermediate in the biosynthesis of different classes of flavonoids including aurones. In this study, two key genes of snapdragon (SRY4'CGT and SRYAS1) in the aurone biosynthetic pathway were cloned, and transformed separately into P. hybrida by an Agrobacteriummediated method. Some transformants carrying the SRY4'CGT or SRYAS1 transgene showed variegated bluewhite flowers (Table 1; Table 2), which were similar to the previous transformants carrying the CHS transgene [25] [26]. A dramatic change in flower color from almost complete blue to variegated blue-white sectors and then complete white in certain transformants carrying SRY4'CGT transgene during plant senescence was also observed (Figure 5). This pattern of alteration in flower coloration has not been previously reported.

Transgene-induced gene silencing is a quite common phenomenon in plants, for example, host genes can be silenced as a consequence of the presence of a homologous transgene [27] [28]. However, in this study, no sequence similarity was found between the host CHS gene and the SRY4'CGT transgene (or between the host CHS gene and the SRYAS1 transgene). It is interesting to find that expression of SRY4'CGT transgene can also suppress the expression of the non-homologous host CHS gene in the white sectors (Figure $8(\mathrm{~d})$ ). On the other hand, suppression of endogenous CHS expression is related to the transgene-induced gene silencing, which is considered to be sequence homology-dependent event [27] [28]. For example, an expression vector pCHS carrying Petunia CHS transgene was transformed into tobacco, nucleotide sequence analysis revealed that both $P e$ tunia and tobacco CHS cDNAs contained 1170 bp in length and sequence identity were 91\%; as a result, four out of seven transgenic plants produced completely white flowers as compared to the pink flowers in wild-type tobacco, and it was further demonstrated that cosuppression of CHS expression resulted the loss of cyanidin pigments in those transgenic white flowers [12]. It has been reported that overexpression of apple anthocyanidin reductase $(A N R)$ genes in tobacco caused inhibition of expression of both chalcone isomerase $(C H I)$ and $D F R$ genes in transgenic flowers, leading to loss of anthocyanin and resulting in white, pale pink and white/red variegated flowers as compared to wild-type red flowers [29]. Since low sequence identity between apple ANS gene and tobacco DFR gene, the authors concluded that cosuppression of tobacco DFR gene might not be related to RNA silencing, and might be due to competition or interaction among different enzymes involved in the flavonoid biosynthetic pathway [29]. Therefore, our transgenic plants should be good material for studying gene expression and regulation in the aurone/flavonoid biosynthetic pathway.

In $\mathrm{T}_{1}$ progeny assay, a number of abnormal segregation ratios (i.e., $\mathrm{R}: \mathrm{S}=1: 1$ or $\mathrm{R}: \mathrm{S}=2: 1$ ) or no seed production was observed (Table 1; Table 2). We noticed that pollen was extremely rare in transgenic SRY4'CGT-1 and SRYAS1-13 plants; this may explain why no seeds were produced in these plants (Table 1; Table 2) (Figure 15). In brief, some of the transgenic plants we obtained in this study seem abnormal. The genus Petunia, belonging to the family Solanaceae, comprises more than 30 species. Most petunias are diploid $(2 n=14)$ and are interfertile with different petunia species. The origin of $P$. hybrida is unknown, but there is general agreement that it was derived from interspecific crossing between a white-flo- wered species (probablyP. axillaris) and a pigmented species (probablyP. integrifolia) near 190 years ago [30]. The complicated genetic background of $P$. hybrid commercial varieties may cause those abnormal phenotypes we observed in this study.

From the point of view of biotechnology application, as the loss of the newly acquired traits in subsequent generations or under certain environmental conditions has been reported elsewhere [6] [31] [32], the next important task is to ensure the reliability and stability of phenotypes reported here after several generations of 

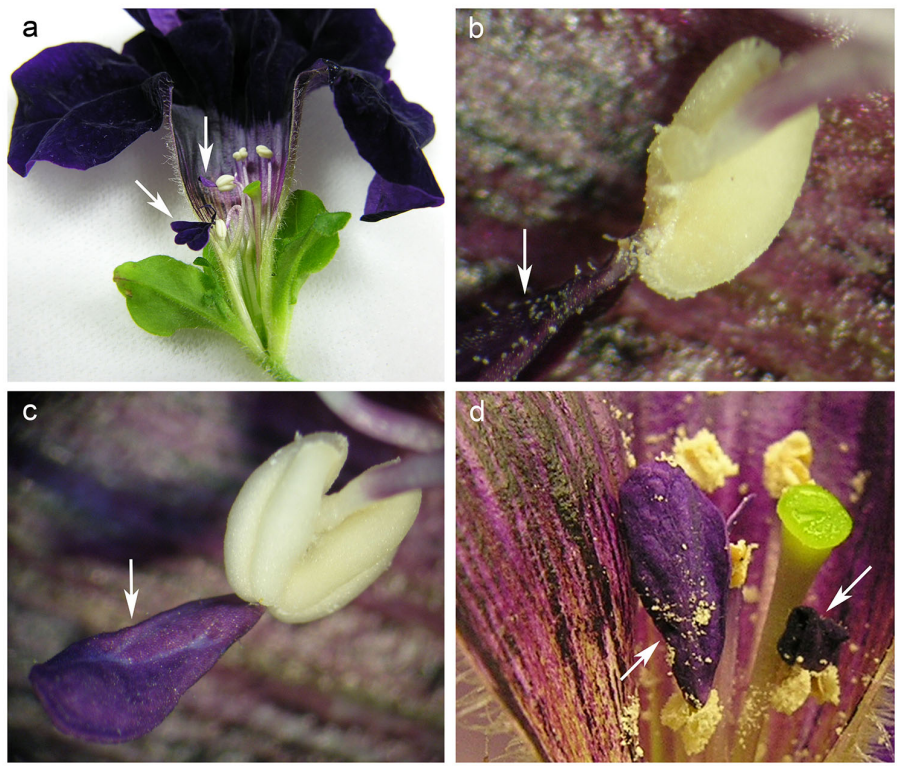

\begin{abstract}
Figure 15. Abnormal flower phenotype showing an extra petal-like structure in two anthers out of five anthers in some transgenic Petunia flowers. (a) Dissection of an abnormal flower. Arrows indicated an extra petal-like structure in two out of five anthers. The fifth anther harboring normal phenotype is not shown in this panel. (b) An enlarged photo of one abnormal anther. The extra petal-like structure appear at the top of the anther. (c) and (d) The abnormal petal-like structure is still presented and even the pollen sac is broken at the late stage of flower development.
\end{abstract}

propagation. Furthermore, it is very important to breed transgenic petunia plant carrying both SRYAS1 and SRY4'- CGT by crossing experiment, which is in progress. In addition, it will be very interesting to examine whether the same phenotypes can be achieved by transforming pSRYAS1 or pSRY4'CGT vector into other floricultural plants, such as Cleome spinosa. Agrobacterium-mediated transformation has been established in this value plant [33].

\title{
Acknowledgements
}

The authors are grateful to the Metabolomics Core Facility, Agricultural Biotechnology Research Center, Academia Sinica, for HPLC-DAD-MS/MS assistance. We also thank Miranda Loney for critical reading and editing of the manuscript. This work was financially supported by Academia Sinica, Taiwan, to Dr. Kin-Ying To.

\section{References}

[1] Bartley, G.E. and Scolnik, P.A. (1995) Plant Carotenoids: Pigments for Photoprotection, Visual Attraction, and Human Health. Plant Cell, 7, 1027-1038. http://dx.doi.org/10.1105/tpc.7.7.1027

[2] To, K.Y. and Wang, C.K. (2006) Molecular Breeding of Flower Color. In: Teixeira da Silva, J.A., Ed., Floriculture, Ornamental and Plant Biotechnology, Volume 1, Global Science Books, London, 300-310.

[3] Nishihara, M. and Nakatsuka, T. (2011) Genetic Engineering of Flavonoid Pigments to Modify Flower Color in Floricultural Plants. Biotechnology Letters, 33, 433-441. http://dx.doi.org/10.1007/s10529-010-0461-z

[4] Schwinn, K.E. and Davies, K.M. (2004) Flavonoids. In: Davies, K.M., Ed., Plant Pigments and Their Manipulations, Blackwell Publishing Ltd., Oxford, 92-149.

[5] Tanaka, Y., Katsumoto, Y., Brugliera, F. and Mason, J. (2005) Genetic Engineering in Floriculture. Plant Cell, Tissue and Organ Culture, 80, 1-24. http://dx.doi.org/10.1007/s11240-004-0739-8

[6] Tanaka, Y., Brugliera, F., Kalc, G., Senior, M., Dyson, B., Nakamura, N., Katsumoto, Y. and Chandler, S. (2010) Flower Color Modification by Engineering of the Flavonoid Biosynthetic Pathway: Practical Perspectives. Bioscience, Biotechnology and Biochemistry, 74, 1760-1769. http://dx.doi.org/10.1271/bbb.100358

[7] Vogt, T. (2010) Phenylpropanoid Biosynthesis. Molecular Plant, 3, 2-20. http://dx.doi.org/10.1093/mp/ssp106 
[8] Chandler, S.F. and Brugliera, F. (2011) Genetic Modification in Floriculture. Biotechnology Letter, 33, 207-214. http://dx.doi.org/10.1007/s10529-010-0424-4

[9] Winkel-Shirley, B. (2001) Flavonoid Biosynthesis: A Colorful Model for Genetics, Biochemistry, Cell Biology, and Biotechnology. Plant Physiology, 126, 485-493. http://dx.doi.org/10.1104/pp.126.2.485

[10] Nakayama, T., Yonekura-Sakakibara, K., Sato, T., Kikuchi, S., Fukui, Y., Fukuchi-Mizutani, M., Ueda, T., Nakao, M., Tanaka, Y., Kusumi, T. and Nishino, T. (2000) Aureusidin Synthase: A Polyphenol Oxidase Homolog Responsible for Flower Coloration. Science, 290, 1163-1166. http://dx.doi.org/10.1126/science.290.5494.1163

[11] Ono, E., Fukuchi-Mizutani, M., Nakamura, N., Fukui, Y., Yonekura-Sakakibara, K., Yamaguchi, M., Nakayama, T., Tanaka, T., Kusumi, T. and Tanaka, Y. (2006) Yellow Flowers Generated by Expression of the Aurone Biosynthetic Pathway. Proceedings of the National Academy of Sciences of the United States of America, 103, 11075-11080. http://dx.doi.org/10.1073/pnas.0604246103

[12] Wang, C.K., Chen, P.Y., Wang, H.M. and To, K.Y. (2006) Cosuppression of Tobacco Chalcone Synthase Using Petunia Chalcone Synthase Construct Results in White Flowers. Botanical Studies, 47, 71-82.

[13] Gerats, T. and Vandenbussche, M. (2005) A Model System for Comparative Research: Petunia. Trends in Plant Science, 10, 251-256. http://dx.doi.org/10.1016/j.tplants.2005.03.005

[14] Lutke, W.K. (2006) Petunia (Petunia hybrid). Methods in Molecular Biology, 344, 339-349.

[15] Murashige, T. and Skoog, F. (1962) A Revised Medium for Rapid Growth and Bioassays with Tobacco Tissue Cultures. Physiologia Plantarum, 15, 473-497. http://dx.doi.org/10.1111/j.1399-3054.1962.tb08052.x

[16] Wang, H.M., Yin, W.C., Wang, C.K. and To, K.Y. (2009) Isolation of Functional RNA from Different Tissues of Tomato Suitable for Developmental Profiling by Microarray Analysis. Botanical Studies, 50, 115-125.

[17] Wang, C.K., Hsu, S.Y., Chen, P.Y. and To, K.Y. (2012) Transformation and Characterization of Transgenic Bidens pilosa L. Plant Cell, Tissue and Organ Culture, 109, 457-464. http://dx.doi.org/10.1007/s11240-011-0110-9

[18] Nakayama, T., Sato, T., Fukui, Y., Yonekura-Sakakibari, K., Hayashi, H., Tanaka, Y., Kusumi, T. and Nishino, T. (2001) Specificity Analysis and Mechanism of Aurone Synthesis Catalyzed by Aureusidin Synthase, a Polyphenol Oxidase Homolog Responsible for Flower Coloration. FEBS Letters, 499, 107-111. http://dx.doi.org/10.1016/S0014-5793(01)02529-7

[19] Giusti, M.M., Rodriguez-Saona, L.E., Griffin, D. and Wrolstad, R.E. (1999) Electrospray and Tandem Mass Spectroscopy as Tools for Anthocyanin Characterization. Journal of Agricultural and Food Chemistry, 47, 4657-4664. http://dx.doi.org/10.1021/jf981242+

[20] Schütz, K., Persike, M., Carle, R. and Schieber, A. (2006) Characterization and Quantification of Anthocyanins in Selected Artichoke (Cynara scolymus L.) Cultivars by HPLC-DAD-ESI-MS ${ }^{\mathrm{n}}$. Analytical and Bioanalytical Chemistry, 384, 1511-1517. http://dx.doi.org/10.1007/s00216-006-0316-6

[21] Hillebrand, S., Naumann, H., Kitzinski, N., Köhler, N. and Winterhalter, P. (2009) Isolation and Characterization of Anthocyanins from Blue-Fleshed Potatoes (Solanum tuberosum L.). Food, 3, 96-101.

[22] Zhao, H., Hu, X., Chen, X., Shi, S., Jiang, X., Chen, W. and Zhang, S. (2015) Analysis and Improved Characterization of Minor Antioxidants from Leaves of Malus doumeri Using a Combination of Major Constituents' Knockout with High-Performance Liquid Chromatography-Diode Array Detector-Quadrupole Time-of-Flight Tandem Mass Spectrometry. Journal of Chromatography A, 1398, 57-65. http://dx.doi.org/10.1016/j.chroma.2015.04.018

[23] Nakatsuka, T., Mishiba, K.I., Kubota, A., Abe, Y., Yamamura, S., Nakamura, N., Tanaka, Y. and Nishihara, M. (2010) Genetic Engineering of Novel Flower Colour by Suppression of Anthocyanin Modification Genes in Gentian. Journal of Plant Physiology, 167, 231-237. http://dx.doi.org/10.1016/j.jplph.2009.08.007

[24] Chandler, S.F. and Sanchez, C. (2012) Genetic Modification; the Development of Transgenic Ornamental Plant Varieties. Plant Biotechnology Journal, 10, 891-903. http://dx.doi.org/10.1111/j.1467-7652.2012.00693.x

[25] Napoli, C., Lemieux, C. and Jorgensen, R. (1990) Induction of a Chimer Chalcone Synthase Gene into Petunia Results in Reversible Co-Suppression of Homologous Genes in trans. Plant Cell, 2, 279-289. http://dx.doi.org/10.1105/tpc.2.4.279

[26] van der Krol, A., Mur, L.A., Beld, M., Mol, J.N.M. and Stuitje, A.R. (1990) Flavonoid Genes in Petunia: Addition of a Limited Number of Gene Copies May Lead to a Suppression of Gene Expression. Plant Cell, 2, 291-299. http://dx.doi.org/10.1105/tpc.2.4.291

[27] Vaucheret, H., Béclin, C., Elmayan, T., Feuerbach, F., Godon, C., Morel, J.-B., Mourrain, P., Palauqui, J.-C. and Vernhettes, S. (1998) Transgene-Induced Gene Silencing in Plants. Plant Journal, 16, 651-659. http://dx.doi.org/10.1046/j.1365-313x.1998.00337.x

[28] Eamens, A., Wang, M.B., Smith, N.A. and Waterhouse, P.M. (2008) RNA Silencing in Plants: Yesterday, Today, and Tomorrow. Plant Physiology, 147, 456-468. http://dx.doi.org/10.1104/pp.108.117275 
[29] Han, Y., Vimolmangkang, S., Soria-Guerra, R.E. and Korban, S.S. (2012) Induction of Apple ANR Genes into Tobacco Inhibits Expression of both CHI and DFR Genes in Flowers, Leading to Loss of Anthocyanin. Journal of Experimental Botany, 63, 2437-2447. http://dx.doi.org/10.1093/jxb/err415

[30] Peltier, D., Farcy, E., Dulieu, H. and Bervillé, A. (1994) Origin, Distribution and Mapping of RAPD Markers from Wild Petunia Species in Petunia hybrida Hort Lines. Theoretical and Applied Genetics, 88, 637-645. http://dx.doi.org/10.1007/BF01253965

[31] Dietz-Pfeilstetter, A. (2010) Stability of Transgene Expression as a Challenge for Genetic Engineering. Plant Science, 179, 164-167. http://dx.doi.org/10.1016/j.plantsci.2010.04.015

[32] Hauser, M.T., Aufsatz, W., Jonak, C. and Luschnig, C. (2011) Transgenerational Epigenetic Inheritance in Plants. Biochimica et Biophysica Acta, 1809, 459-468. http://dx.doi.org/10.1016/j.bbagrm.2011.03.007

[33] Tsai, Y.T., Chen, P.Y. and To, K.Y. (2012) Plant Regeneration and Stable Transformation in the Floricultural Plant Cleome spinosa, a $\mathrm{C}_{3}$ Plant Closely Related to the $\mathrm{C}_{4}$ Plant C. gynandra. Plant Cell Reports, 31, 1189-1198. http://dx.doi.org/10.1007/s00299-012-1240-1 